\title{
A Prokaryotic Membrane Sculpting BAR Domain Protein
}

Daniel A. Phillips ${ }^{1 *}$, Lori A. Zacharoff ${ }^{*}$, Cheri M. Hampton ${ }^{3}$, Grace W. Chong ${ }^{4}$, Anthony P. Malanoski ${ }^{5}$, Lauren Ann Metskas ${ }^{6}$, Shuai $\mathrm{Xu}^{2}$, Lina J. Bird ${ }^{5}$, Brian J. Eddie ${ }^{5}$, Grant J. Jensen $^{6,7}$, Lawrence F. Drummy ${ }^{3}$, Mohamed Y. El-Naggar ${ }^{2,4,8}$, and Sarah M. Glaven ${ }^{5}$

${ }^{1}$ American Society for Engineering Education, Washington, DC, USA.

${ }^{2}$ University of Southern California, Department of Physics and Astronomy, Los Angeles, CA 90089

${ }^{3}$ Materials and Manufacturing Directorate, Air Force Research Laboratory, WrightPatterson Air Force Base, WPAFB, OH, USA.

${ }^{4}$ University of Southern California, Department of Biological Sciences, Los Angeles, CA 90089

${ }^{5}$ Center for Bio/Molecular Science and Engineering, Naval Research Laboratory, Washington, DC, USA.

${ }^{6}$ California Institute of Technology, Division of Biology and Biological Engineering, Pasadena, CA 91125

${ }^{7}$ California Institute of Technology, Howard Hughes Medical Institute, Pasadena, CA 91125

${ }^{8}$ University of Southern California, Department of Chemistry, Los Angeles, CA 90089

"These authors contributed equally to this work

Classification: Biological sciences

Keywords: BAR domain | extracellular electron transport | membrane shaping | outer membrane vesicles | outer membrane extensions | Shewanella

\section{Send all correspondence to:}

Daniel A. Phillips

Daniel.a.phillips62.ctr@mail.mil

(423) 215-2256

US Army CCDC CBC, Building E3549

Aberdeen Proving Grounds Edgewood Area

Gunpowder, MD 21010

Lori A. Zacharoff

zacharof@usc.edu

(763) 221-0960

920 Bloom Walk

SSC, 215C

Los Angeles, CA 90089 


\section{Abstract}

Bin/Amphiphysin/RVS (BAR) domain proteins belong to a superfamily of coiled-

3 coil proteins influencing membrane curvature in eukaryotes and are associated with

4 vesicle biogenesis, vesicle-mediated protein trafficking, and intracellular signaling.

5 Here we report the first prokaryotic BAR domain protein, BdpA, from Shewanella oneidensis MR-1, known to produce redox-active membrane vesicles and micrometerscale outer membrane extensions (OMEs). BdpA is required for uniform size distribution

8 of membrane vesicles and scaffolding OMEs into a consistent diameter and curvature.

9 Cryogenic transmission electron microscopy reveals a strain lacking BdpA produces

10 lobed, disordered OMEs rather than membrane tubes produced by the wild type strain.

11 Overexpression of BdpA promotes OME formation during conditions where they are

12 less common. Heterologous expression results in OME production in Marinobacter atlanticus and Escherichia coli. Based on the ability of BdpA to alter membrane

14 curvature in vivo, we propose that BdpA and its homologs comprise a newly identified 15 class of prokaryotic BAR (P-BAR) domains. 


\section{Introduction}

Eukaryotic Bin/Amphiphysin/Rvs (BAR) domain-containing proteins generate membrane curvature through electrostatic interactions between positively charged amino acids and negatively charged lipids, scaffolding the membrane along the intrinsically curved surface of the antiparallel coiled-coil protein dimers (1-4). Some BAR domaincontaining proteins, such as the N-BAR protein BIN1, contain amphipathic helical wedges that insert into the outer membrane leaflet and can assist in membrane binding (5). Other BAR domains can be accompanied by a membrane targeting domain, such as PX for phosphoinositide binding $(6,7)$, in order to direct membrane curvature formation at specific sites, as is the case with sorting nexin BAR proteins (8). The extent of accumulation of BAR domain proteins at a specific site can influence the degree of the resultant membrane curvature (9), and tubulation events arise as a consequence of BAR domain multimerization in conjunction with lipid binding (10). Interactions between BAR domain proteins and membranes resolve membrane tension, promote membrane stability, and aid in localizing cellular processes, such as actin binding, signaling through small GTPases, membrane vesicle scission, and vesicular transport of proteins (11-13). Despite our knowledge of numerous eukaryotic BAR proteins spanning a variety of modes of curvature formation, membrane localizations, and subtypes (N-BAR, F-BAR, and I$B A R)$, characterization of a functional prokaryotic BAR domain protein has yet to be reported.

Bacterial cell membrane curvature can be observed during the formation of outer membrane vesicles (OMV) and outer membrane extensions (OME). OMV formation is ubiquitous and has many documented functions (14). OMEs are less commonly 
observed, remain attached to the cell, and various morphologies can be seen extending

41 from single cells including Myxococcus xanthus $(15,16)$, flavobacterium strain

42 Hel3_A1_48 (17), Vibrio vulnificus (18), Francisella novicida (19), Shewanella oneidensis

43 (20-23), and as cell-cell connections in Bacillus subtilis (24-26) and Escherichia coli (27).

44 Several bacterial proteins have demonstrated membrane tubule formation capabilities in

45 vitro (28-33), but despite the growing number of reports, proteins involved in shaping bacterial membranes into OMV/Es have yet to be identified. Recently, researchers have

47 begun to suspect that OMV and OME formation has some pathway overlap (17), and it is proposed that proteins are necessary to stabilize these structures (34).

Shewanella oneidensis is a model organism for extracellular electron transfer (EET), a mode of respiration whereby electrons traverse the inner membrane, periplasm,

51 and outer membrane via multiheme cytochromes to reach exogenous insoluble terminal

52 electron acceptors, such as metals and electrodes $(35,36)$. S. oneidensis is known to

53 produce redox-active OMVs (37) and OMEs coated with mulitheme cytochromes, 54 particularly upon surface attachment $(20,22,37)$. However, little is known about their formation mechanism, control of shape or curvature, and electrochemical properties that 56 influence EET function. Previously, OMEs of S. oneidensis were shown to transition between chains of vesicles and tubules. We identified a component critical to this structural transition as the first BAR domain protein in prokaryotes, which we term BdpA (BAR domain-like protein A). Through comparative proteomics, cryogenic electron microscopy, and molecular biology, we show that BdpA is enriched in OMENs, regulates size of OMVs, and controls the shape of OMEs. Likewise, BdpA confers OME formation capabilities when expressed 
63 in other bacteria, showing mechanistic evidence of BAR domain protein-mediated tubule

64 formation in vivo. This study provides a framework for prokaryotic BAR domain characterization, and putative BAR domain-containing BdpA homologs in other bacteria

66 suggest BAR domain protein-mediated membrane sculpting is an evolutionarily

67 conserved function.

\section{Results and Discussion}

\section{S. oneidensis OMVs are redox-active and enriched with BdpA}

OMVs were purified from $S$. oneidensis cells grown in batch cultures to characterize their redox features and unique proteome, as well as to identify putative

72 membrane shaping proteins. Cryogenic transmission electron microscopy (cryo-TEM) tomographic reconstruction slices of the purified samples showed uniform OMVs with the characteristic single membrane phenotype and an approximate diameter of $200 \mathrm{~nm}$ (Fig.

1a). Previous measurements showed OMVs can reduce extracellular electron acceptors (37) and that vesicles from G. sulfurreducens can mediate electron transfer (38). Electrochemical activity of multiheme cytochrome complex MtrCAB and their ability to mediate micrometer-scale electron transport has been characterized in whole cells

79 (39), but no electrochemical characterization of OME/Ns has been reported that link activity to multiheme cytochromes. Here, electrochemical measurements of isolated

81 OMVs were performed to determine if purified OMVs maintain the characteristic redox

82 features of the MtrCAB complex when detached from cells. Cyclic voltammetry (CV) 83 demonstrated redox activity of isolated membrane vesicles adhered to a gold electrode 84 via self-assembled monolayers (Fig. 1b). The first derivative (Fig. 1b inset) revealed a 
a standard hydrogen reference electrode (SHE). This midpoint potential is consistent with

87 the characteristics of multiheme cytochromes such as MtrC/OmcA from previous microbial electrochemical studies $(39,40)$, suggesting that the extracellular redox molecules of the cellular outer membrane extends to OMVs.

The proteome of the OMVs was compared to the proteome of purified outer membranes extracted from whole cells. Using a label-free quantification method (41), significant differences in the ratio of individual proteins in the vesicle to the outer membrane could be computed (log fold change) (Fig 1c). The proteome of the purified OMVs showed 328 proteins were significantly enriched in the vesicles as compared to the outer membrane, and 314 proteins were significantly excluded from the vesicles (Fig. 1c, Supplemental table 1). MtrCAB cytochromes were present in the OMVs as well as the outer membrane, supporting redox activity of OMVs observed by CV. Active protein sorting into eukaryotic vesicles is a coordinated process involving protein sorting signal recognition, localized membrane protein recruitment, initiation of membrane curvature induction, and coating nascent vesicles with protein scaffolds (42). Several proteins significantly enriched in the vesicles were identified that could contribute to OMV formation, including the murein transglycosylase, the peptidoglycan degradation enzyme holin, cell division coordinator $\mathrm{CpoB}$, and a highly enriched putative BAR domaincontaining protein encoded by the gene at open reading frame SO_1507, hereafter named BAR domain-like protein A (BdpA) (Fig. 1d).

Vesicle enrichment of BdpA led us to the hypothesis that BdpA could be involved in membrane shaping of OMVs based on the role of BAR domain proteins in eukaryotes. The C-terminal BAR domain of BdpA is predicted to span an alpha-helical region from AA 
$276-451(E-v a l u e=2.96 e-03)$; however, since the identification of the protein is based on homology to the eukaryotic BAR domain consensus sequence (cd07307), it is possible that the BAR domain region extends beyond these bounds (Fig. 1d). Coiled coil prediction (43) suggests BdpA exists in an oligomeric state of antiparallel alpha-helical dimers, as is the case for all known BAR domain proteins $(2,44-46)$. The predicted structure of the BAR domain-containing region generated in i-TASSER (47) shows a 3 helix bundle (Supplemental Fig. 1a). When the BdpA BAR domain monomers were aligned to the dimeric structure of the F-BAR protein Hof1p (48), the predicted dimer interface residues of BdpA came into proximity, revealing an intrinsically curved dimer with positively charged residues along the concave surface (Supplemental Fig. 1b). BdpA has a Nterminal signal peptide with predicted cleavage sites between amino acids 22-23, suggesting non-cytoplasmic localization (Fig. 1d). A galactose-binding domain-like region positioned immediately downstream of the signal peptide supports lipid targeting activity seen in other BAR domain proteins, such as the eukaryotic sorting nexins (49) which have phox (PX) domains that bind phosphoinositides (50). The S. oneidensis rough-type lipopolysaccharide (LPS) contains 2-acetamido-2-deoxy-D-galactose (51), which suggests possible localization of the protein to the outer leaflet of the outer membrane.

\section{BdpA controls size distribution of vesicles}

In eukaryotic cells, BAR domain proteins are implicated in vesicle formation (52, 53) and regulation of vesicle size (54). To determine whether BdpA influences vesicle morphology, OMVs were harvested from wild type (WT) cells and cells in which the gene for BdpA had been deleted $(\triangle b d p A)$, and their diameters were measured by dynamic light 
132 scattering (DLS). WT OMVs had a median diameter of $190 \mathrm{~nm}$ with little variability in the population (standard deviation (s.d.) $= \pm 21 \mathrm{~nm}$ ), while the diameters of $\triangle b d p A$ OMVs were distributed over a wider range with a median value of $280 \mathrm{~nm}$ (s.d. $= \pm 131 \mathrm{~nm}$ (Fig 2a).

135 The data suggest BdpA controls vesicle diameter in membrane structures ex vivo, 136 potentially acting by stabilizing OMVs. cultures using a perfusion flow imaging platform and the membrane stain FM 4-64, as described previously (21). S. oneidensis strains were monitored for OMEN production and progression over the course of 5 hours ( $>5$ fields of view per replicate, $n=3$ ) using

141 time-lapse imaging. Spherical membrane stained extracellular structures were classified 142 as OMVs, while larger aspect ratio (i.e. length greater than the width) structures were 143 classified as OMEs. The proportion of cells producing 'large' vesicles, defined as having a membrane clearly delineated from the interior of the vesicles and typically $>300 \mathrm{~nm}$, was quantified, and $\Delta b d p A$ cells produced significantly more large vesicles compared to 146 WT cells (Fig. 2b) even though both the overall frequency of vesiculation and extensions 147 were the same (Fig. 2c). Previous studies showed that OMEs transition between large 148 vesicles and OMEs over time (21). BdpA appears to be involved in this transition in 149 Shewanella due to the increased frequency of large vesicles from $\Delta b d p A$ cells. The 150 median diameter of the OMVs is also the apparent maximum diameter observed in outer 151 membrane extensions (21) suggesting BdpA influences membrane morphologies of both 152 structures. 


\section{BdpA constrains membrane extension morphology}

Cells were also visualized after deposition onto a glass coverslip instead of a perfusion flow chamber as previously reported (20). BdpA was expressed from a 2,4diacetylphloroglucinol (DAPG)-inducible promoter (55) (PphlF-BdpA) in the $\Delta b d p A$ strain containing the plasmid p452-bdpA with $12.5 \mu \mathrm{M}$ DAPG. After 3 hours post deposition on cover glass, OMEs can be seen extending from WT, $\triangle b d p A$, and $\triangle b d p A p 452-b d p A$ cells (Fig. 3a, Supplemental Fig. 2, Supplemental videos 1-3, 5 fields of view, n=3). Similar to perfusion flow experiments, no statistically significant difference in the overall frequency of OME production was observed between the cells in static cultures. The resolution of fluorescence microscopy was insufficient to identify morphological differences between OMEs of wild type and mutant strains, therefore, cryo-TEM was used to assess OMEs in each of the strains at the ultrastructural level.

S. oneidensis OMEs from unfixed WT, $\triangle b d p A$, and $\triangle b d p A$ p452-bdpA strains were visualized at 90 minutes (Supplemental Fig. 3) and 3 hours (Fig. 3a) following cell deposition onto EM grids. By 3 hours post inoculation, images of WT cells consistently show narrow, tubule-like OMEs or vesicle chains of symmetric curvature (Fig. 3b,c n=31 OMEs observed). The $\triangle b d p A$ OMEs appear as lobed, disordered vesicle chains with irregular curvature compared to the WT ( $p<0.001$, Fisher's exact test), and vesicles can be observed branching laterally from lobes on the extensions (Fig. 3b,c $n=13$ OMEs observed). WT OMEs also exhibited lateral branching of vesicles and lobes, but they exhibited uniform curvature and diameter between lobes, mirroring previous observations of nascent OMEs imaged immediately following OME formation (21). Tubules were not observed in any $\triangle b d p A$ OMEs at 3 hours (Fig. $3 d$ ). OMEs from $\triangle b d p A$ p452-bdpA cells 
176 appear as narrow tubules of a uniform curvature or as ordered vesicle chains (Fig. 3b,c

$177 \mathrm{n}=3$ OMEs observed), showing that expression of BdpA from a plasmid rescues the

178 mutant phenotype by constricting and ordering OMEs into narrow tubules and chains (Fig.

$1793 d, p<0.01$, Fisher's exact test). Earlier in OME progression at 90 minutes, WT OME

180 phenotypes appeared narrow, tubule-like, and seldom interspersed with lobed regions

181 (Supplemental figure 3a). In $\triangle b d p A$ OMEs, lobed regions are prevalent with irregular

182 curvature (Supplemental figure 3b). Several narrow $\triangle b d p A$ p452-bdpA OMEs evenly

183 interspersed with slight constriction points or "junction densities" were observed extending

184 from a single cell (Supplemental Fig. 3c).

185 Expression of BdpA results in OMEs during planktonic growth

S. oneidensis OMEs are more commonly observed in surface attached cells than planktonic cells $(20,21)$. BAR domain proteins can directly promote tubule formation from liposomes in vitro(9), so inducing expression of an additional copy of the $b d p A$ gene prior to attachment could result in OME formation even during planktonic growth. Growth curves were similar in cultures with the pBBR1-mcs2 empty vector in either of the WT or $\triangle b d p A$ background strains, but induction of $b d p A$ in $\triangle b d p A$ p452-bdpA cells at higher concentrations of 1.25 and $12.5 \mu \mathrm{M}$ 2,4-diacetylphloroglucinol affected the growth rate 193 (Supplemental figure 4). Planktonic cultures inoculated from overnight cultures were 194 induced with 12.5 $\mu$ M DAPG for 1 hour, labeled with FM 4-64, and imaged by confocal 195 microscopy. Neither WT (Fig. 4) nor WT with the empty plasmid exposed to $12.5 \mu \mathrm{M}$ DAPG (not shown) displayed OMEs immediately following deposition onto cover glass.

197 However, $12.5 \mu \mathrm{M}$ DAPG-induced bdpA expression from $\mathrm{p} 452-b d p A$ in the WT background strain (WT p452-bdpA) displayed OMEs immediately, ranging between 1-7 
extensions per cell (Figure 4, Supplemental video 4). The abundance of OMEs suggest that increased $b d p A$ expression in planktonic cultures can initiate membrane sculpting into OMEs at the expense of cell division.

The ultrastructure of OMEs resulting from expression of $b p d A$ from WT p452-bdpA cells was examined by cryo-TEM, but in this case samples from planktonic cultures were vitrified on EM grids after induction rather than incubation during induction on the EM grids. By expressing extra copies of $b d p A$ in the WT strain during planktonic induction, we predicted that OME morphology would be predominately reliant on curvature formation by BdpA rather than other unidentified structural proteins involved in intrinsic OME formation. OMEs appear as tubule-like segments interspersed with pearled regions proximal to the main cell body (Fig. 4b). OMEs from the MR-1 p452-bdpA strain are observed as thin, tubule-like outer membrane vesicle chains, suggesting BdpA involvement in the constriction of the larger outer membrane vesicle chains into longer, tubule-like extensions with more evenly interspersed junction densities. The BdpA OME phenotype more closely resembles membrane tubules formed by the F-BAR protein Pacsin1 from eukaryotic cells, showing a mixture of tubule regions interspersed with pearled segments $(56,57)$. Orientation and association of Pacsin1 dimers on the membrane surface with one another impacted tubule morphology, and BdpA membrane sculpting could be similar mechanistically.

BdpA-mediated membrane extensions in Marinobacter atlanticus CP1 and E. coli. To test the effect of expressing BdpA in an organism with no predicted BAR domain-containing proteins and no apparent OME production, BdpA was expressed in Marinobacter atlanticus CP1 (58). Marinobacter and Shewanella are of the same 
222 phylogenetic order (Alteromonadales) and have been used for heterologous expression

223 of other $S$. oneidensis proteins, such as $\operatorname{MtrCAB}(59,60)$. Upon exposure to DAPG, $M$.

224 atlanticus containing the $\mathrm{p} 452-b d p A$ construct (CP1 p452-bdpA) forms membrane

225 extensions (Figure 4). OMEs ranged from small membrane blebs to OME tubules

226 extending up to greater than $10 \mu \mathrm{m}$ in length from the surface of the cell (Supplemental

227 Fig. 5). As noted previously, variation in the tubule phenotypes are commonly seen in

228 tubules from eukaryotic F-BAR proteins $(56,57)$, showing possible mechanistic overlap

229 of membrane curvature functionalities between these two separate BAR domain proteins.

In previous membrane curvature formation experiments with eukaryotic BAR

231 domain proteins, localized BAR domain protein concentrations affected the resultant

232 shape of the membranes, ranging from bulges to tubules and branched, reticular tubule

233 networks at the highest protein densities (61-63). We predicted that expression of BdpA

234 in cells optimized for protein overexpression, such E. coli BL21(DE3), would show OMEs

235 resembling BAR protein concentration-dependent structures previously observed from

236 eukaryotic BAR protein experiments in vitro. While the uninduced $E$. coli BL21(DE3)

$237 \mathrm{p} 452-b d p A$ cells had uniform, continuous cell membranes similar to those of plasmid-free

238 BL21(DE3) cells under the conditions tested, E. coli BL21(DE3) cells containing the p452-

$239 \quad b d p A$ vector induced with $12.5 \mu \mathrm{M}$ DAPG had outer membrane extensions and vesicles

240 (Figure 4). When visualized over time, OMEs progressed towards a network of reticular

241 membrane structures extending from the cell (Fig. 4c). After 30 minutes, additional

242 membrane blebs were observed that developed into elongated OMEs by 60 minutes.

243 Growth of E. coli OMEs was coincident with shrinking of the cell body (from initial cell

244 length $=4.5 \mu \mathrm{m}$ to $3.5 \mu \mathrm{m}$ at 60 minutes), supporting direct membrane sculpting activity 
245 of BdpA. In vitro tubule formation assays with purified proteins and liposomes are the canonical approach by which eukaryotic BAR domain proteins have been assessed for membrane sculpting activity. However, molecular crowding of purified proteins with no documented membrane curvature formation activity, such as GFP, can also lead to ordering of liposomes into tubules (64). Further, tubule formation from liposomes is not

250 limited to BAR domain protein activity and requires non-physiologically high protein 251 concentrations (65-67). The heterologous expression approach allows for more rapid 252 screening of putative BAR domain proteins for membrane sculpting activity, avoiding the need for protein purification and in vitro systems.

\section{P-BAR: a new BAR domain subtype}

were identified by PSI-BLAST in other Gammaproteobacteria, including most but not all species of Shewanella, as well as Alishewanella, Rheinheimera, and Cellvibrio

261 (Supplemental Fig. 6). The current BAR domain Pfam Hidden Markov Model (HMM) prediction analysis identified BAR domain features in only 5 of the 52 prokaryotic alignment of the $52 \mathrm{BdpA}$ homologs was used to generate a maximum likelihood

266 prediction sequences (Supplemental Fig. 6). The 5 BdpA orthologs predicted to contain 
268 known BAR proteins from the various BAR domain subtypes (N-BAR, F-BAR, and IBAR)(68). BdpA and its prokaryotic orthologs cluster separately from the eukaryotic BAR proteins in their own distinct clade (Fig. 5), suggesting that while BdpA contains a

271 functional BAR domain, it represents its own class of BAR domain, hereafter named P-

272 BAR (Prokaryotic BAR). The closest phylogenetic relative to P-BAR domains are the F-

273 BAR domains, and BdpA membrane sculpting phenotypes are functionally similar to 274 previous in vitro observations from Pacsin1 $(56,57)$. It is possible that the P-BAR domain 275 arose as a result of horizontal gene transfer from a eukaryote due to the prevalence of 276 eukaryotic coiled-coil proteins with predicted homology to BdpA after 2 iterations of PSI-

277 BLAST. However, the branch lengths and low bootstrap values supporting the placement of P-BAR relative to other BAR domain subtypes make it challenging to directly infer the evolutionary history of P-BAR domains. Discovery of other putative P-BAR proteins would help to build this analysis, and if future comparative proteomics analysis of OMENs demonstrates overlapping activity of BdpA with preferential cargo loading into OME/Vs, it could hint at the evolutionary origins of vesicle-based protein trafficking. Conservation of BAR domain proteins supports the notion that three-dimensional organization of proteins in lipid structures is as important to prokaryotes as it is eukaryotes, and suggests

285 additional novel P-BAR proteins are waiting to be discovered.

288 first identified and characterized in bacteria. Enrichment of BdpA in the redox-active 289 OMVs suggests overlapping mechanistic functionality with eukaryotic BAR proteins in the 290 context of vesicle constriction (52). This finding was further demonstrated through 
291 fluorescence microscopy during perfusion flow, where large vesicles were more 292 frequently observed from $\Delta b d p A$. Membrane constriction activity of BdpA was confirmed

293 through cryo-EM images that depicted an inability to transition into ordered tubules in the 294 absence of $b d p A$ expression. Variation in OMEs with BdpA ranged from ordered, narrow 295 vesicle chains of a consistent diameter to stable tubules. The closest phylogenetic 296 eukaryotic BAR domain subtype to BdpA, F-BAR domains, exhibit similar variation in 297 tubule morphology, depending upon the orientation of the tip-to-tip oligomerization around 298 the tubules $(2,3,57)$. Subsequent studies will include vesicle constriction into tubules 299 with purified protein to ascertain the extent of functional mechanistic similarity of BdpA to 300 other F-BAR proteins. However, heterologous expression of BdpA and other potential P301 BAR domain proteins enables rapid validation of membrane sculpting mechanistic 302 activity. Ultimately, the discovery of BdpA and its homologs presents a critical first step in 303 the new field of bacterial BAR domain protein research. 


\section{Methods}

Bacterial strains, plasmids, and medium The bacterial strains used in this study can

be found in Supplemental Table 1. S. oneidensis strains were grown aerobically in Luria

Bertani (LB) media at $30^{\circ} \mathrm{C}$ with $50 \mu \mathrm{g} / \mathrm{mL}$ kanamycin when maintaining the plasmid. To observe membrane extensions, cells were centrifuged and resuspended in a defined media comprised of $30 \mathrm{mM}$ Pipes, $60 \mathrm{mM}$ sodium DL-lactate as an electron donor, $28 \mathrm{mM}$ $\mathrm{NH}_{4} \mathrm{Cl}, 1.34 \mathrm{mM} \mathrm{KCl}, 4.35 \mathrm{mM} \mathrm{NaH}_{2} \mathrm{PO}_{4}, 7.5 \mathrm{mM} \mathrm{NaOH}, 30 \mathrm{mM} \mathrm{NaCl}, 1 \mathrm{mM} \mathrm{MgCl} 2,1$ $\mathrm{mM} \mathrm{CaCl}_{2}$, and $0.05 \mathrm{mM}$ ferric nitrilotriacetic acid(22). Marinobacter atlanticus CP1 strains were grown in BB media (50\% LB media, 50\% Marine broth) at $30^{\circ} \mathrm{C}$ with 100 $\mu \mathrm{g} / \mathrm{mL}$ kanamycin to maintain the plasmids as described previously (58). MR-1, M. atlanticus CP1, and E. coli BL21(DE3) using the pBBR1-mcs2 backbone described previously (58). The Marionette sensor components (phlF promoter, consitutively expressed PhIF repressor, and yellow fluorescence protein (YFP)) cassette

318 from pAJM.452 (55) was cloned into the pBBR1-mcs2 backbone, and the YFP cassette was replaced with the gene encoding $\mathrm{BdpA}$ by Gibson assembly (primers in Supplemental Table 2). The resulting plasmid was given the name p452-bdpA. The

321 Gibson assembly reactions were electroporated into E. coli Top10 DH5a cells 322 (Invitrogen), and the sequences were confirmed through Sanger sequencing (Eurofins 323 genomics). Plasmid constructs were chemically transformed into conjugation-competent

324 E. coli WM3064 cells for conjugative transfer into the recipient bacterial strains of $S$. 325 oneidensis MR-1 and $M$. atlanticus CP1. The same BdpA expression vector was 326 transformed into E. coli BL21(DE3) cells (Invitrogen) by chemical transformation. 
was transformed into E. coli DH5a Apir strain UQ950 cells for propagation. Plasmid sequences were confirmed by Sanger sequencing before chemical transformation into $E$. coli WM3064 for conjugation into S. oneidensis. Conjugation of pSMV3_1507KO into S. oneidensis MR-1 was performed as described previously (23). Optical densities at 600nm were measured to determine growth curves for each strain in either LB or SDM with 50 $\mu \mathrm{g} / \mathrm{mL}$ kanamycin and 2,4-diacetylphloroglucinol (DAPG) as indicated. Cultures of 500 plates (Corning Incorporated) within a Tecan Infinite M1000 Pro (Grödig, Austria) plate reader at $30^{\circ} \mathrm{C}$ with shaking agitation at $258 \mathrm{rpm}$. Optical densities were recorded every 15 minutes with i-Control software (2.7). All measurements were performed in triplicate.

Purification of Outer Membrane Vesicles S. oneidensis MR-1 cells were grown in LB in $1 \mathrm{~L}$ non-baffled flasks at $30^{\circ} \mathrm{C}$ at $200 \mathrm{RPM}$. When an OD600 of 3.0 was reached, cells were pelleted by centrifugation at $5000 \times \mathrm{g}$ for $20 \mathrm{~min}$ at $4^{\circ} \mathrm{C}$, resulting supernatant was 343 filtered through a $0.45 \mu \mathrm{m}$ filter to remove remaining bacterial cells. Vesicles were 344 obtained by centrifugation at $38,400 \times \mathrm{g}$ for $1 \mathrm{~h}$ at $4^{\circ} \mathrm{C}$ in an Avanti $\mathrm{J}-20 \mathrm{XP}$ centrifuge 345 (Beckman Coulter, Inc). Pelleted vesicles were resuspended in $20 \mathrm{ml}$ of $50 \mathrm{mM}$ HEPES $346(\mathrm{pH} 6.8)$ and filtered through $0.22 \mu \mathrm{m}$ pore size filters. Vesicles were again pelleted as 347 described above and finally resuspended in $50 \mathrm{mM} \mathrm{HEPES}, \mathrm{pH}$ 6.8, except for vesicle preparations used for electrochemistry which were suspended in 100 mM MES, 100 mM 
$349 \mathrm{KCl}, \mathrm{pH}$ 6.8. Extracellular DNA, flagella, and pili can all be co-purified. Protocol was 350 adapted from Perez-Cruz et al (69).

351 Cryoelectron tomography Vesicle samples were diluted to a protein concentration of $3520.4 \mathrm{mg} / \mathrm{mL}$ and applied to glow-discharged, X-thick carbon-coated, R2/2, 200 mesh 353 copper Quantifoil grid (Quantifoil Micro Tools) using a Vitrobot chamber (FEl). Grids were automatically plunge frozen and saved for subsequent imaging. No fixative was used. Images were collected on an FEI Krios transmission electron microscope equipped with a K2 Summit counting electron-detector camera (Gatan). Data were collected using customized scripts in SerialEM(70), with each tilt series ranging from $-60^{\circ}$ to $60^{\circ}$ in $3^{\circ}$ 358 increments, an underfocus of $\sim 1-5 \mu \mathrm{m}$, and a cumulative electron dose of $121 \mathrm{e} / \mathrm{A}^{2}$ for each individual tilt series. Tomograms were reconstructed using a combination of ctffind4 (71) and the IMOD software package (72).

Dynamic Light Scattering Distributions of vesicle diameters were measured with Wyatt

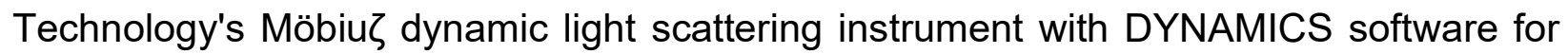
data collection and analysis. Data was collected using a 0-50 mW laser at $830 \mathrm{~nm}$. The scattered photons were detected at $90^{\circ}$. Measurements were recorded from 11 biological replicates for WT OMVs, and 9 replicates for $\triangle b d p A$ OMVs. Mobius software analyzed the population of particles to generate a table with binned diameters and the percentage 368 of particles at each diameter. In order to compute the average vesicle size from each sample, a weighted average was computed so that diameter bins that had the most

370 number of vesicles would be accurately represented in the final average weight of the 371 population. The product of each diameter is multiplied by its percentage in the population, 372 these products are added together for each sample, divided by the sum of weights. The 
373 weighted diameters per replicate were then averaged for each genotype. Statistical 374 significance was determined by Student's $t$-test, and error bars represent standard 375 deviation.

376 Electrochemistry CHA Industries Mark 40 e-beam and thermal evaporator was used to 377 deposit a $5 \mathrm{~nm} \mathrm{Ti}$ adhesion layer and then a $100 \mathrm{~nm}$ Au layer onto cleaned glass coverslips (43mm x 50mm \#1 Thermo Scientific Gold Seal Cover Glass, Portsmouth NH,

379 USA). Self-assembled monolayers were formed by incubated the gold coverslip in a 380 solution of $1 \mathrm{mM} 6$-mercaptohexanoic acid in 200 proof ethanol for at least 2 hours.

381 Electrode was then rinsed several time in ethanol followed by several rinses in milliQ 382 water. The SAMs layer was then activated by incubation in $100 \mathrm{mM} \mathrm{N}-(3-$ 383 Dimethylaminopropyl)-N'-ethylcarbodiimide hydrochloride and $25 \quad \mathrm{mM} \quad \mathrm{N}-$ 384 hydroxysuccinimide, $\mathrm{pH} 4$, for 30 minutes. A sample of outer membrane vesicles was 385 deposited on the surface of the electrode and incubated at room temperature overnight 386 in a humid environment. Cyclic voltammetry was performed in a $50 \mathrm{~mL}$ three-electrode 387 half-cell completed with a platinum counter electrode, and a $1 \mathrm{M} \mathrm{KCl} \mathrm{Ag} / \mathrm{AgCl}$ reference 388 electrode electrical controlled by a Gamry 600 potentiostat (Gamry, Warminster, PA). 389 The whole experiment was completed in an anaerobic chamber with $95 \%$ nitrogen, $5 \%$ 390 hydrogen atmosphere. 
391 Proteomics Vesicle samples were prepared as described above. S. oneidensis outer 392 membrane (OM) was purified via the Sarkosyl method described by Brown et al.(73). A $50 \mathrm{~mL}$ overnight culture of cells was harvested by centrifugation at $10,000 \times \mathrm{g}$ for $10 \mathrm{~min}$.

394 The cell pellet suspended in $20 \mathrm{~mL}$ of $20 \mathrm{mM}$ ice-cold sodium phosphate $(\mathrm{pH} 7.5)$ and 395 passed four times through a French Press $\left(12000 \mathrm{lb} / \mathrm{in}^{2}\right)$. The lysate was centrifuged at $3965,000 \times \mathrm{g}$ for $30 \mathrm{~min}$ to remove unbroken cells. The remaining supernatant was 397 centrifuged at $45,000 \times \mathrm{g}$ for $1 \mathrm{~h}$ to pellet membranes. Crude membranes were 398 suspended in $20 \mathrm{~mL}$ 0.5\% Sarkosyl in $20 \mathrm{mM}$ sodium phosphate and shaken horizontally 399 at $200 \mathrm{rpm}$ for $30 \mathrm{~min}$ at room temperature. The crude membrane sample was centrifuged at $45,000 \times \mathrm{g}$ for $1 \mathrm{~h}$ to pellet the OM. The pellet of OM was washed in ice-cold sodium 401 phosphate and recentrifuged.

402

To prepare for mass spectrometry samples were treated sequentially with urea,

403 TCEP, iodoactinamide, Iysl endopeptidase, trypsin, and formic acid. Peptides were then 404 desalted by HPLC with a Microm Bioresources C8 peptide macrotrap (3x8mm). The 405 digested samples were subjected to LC-MS/MS analysis on a nanoflow LC system, 406 EASY-nLC 1200, (Thermo Fisher Scientific) coupled to a QExactive HF Orbitrap mass 407 spectrometer (Thermo Fisher Scientific, Bremen, Germany) equipped with a Nanospray 408 Flex ion source. Samples were directly loaded onto a PicoFrit column (New Objective, 409 Woburn, MA) packed in house with ReproSil-Pur C18AQ 1.9 um resin (120A pore size, 410 Dr. Maisch, Ammerbuch, Germany). The $20 \mathrm{~cm}$ x $50 \mu \mathrm{m}$ ID column was heated to $60^{\circ}$ 411 C. The peptides were separated with a 120 min gradient at a flow rate of $220 \mathrm{~nL} / \mathrm{min}$.

412 The gradient was as follows: 2-6\% Solvent B (7.5 min), 6-25\% B (82.5 min), and 25-40\% 413 B (30 min) and to $100 \%$ B (9min). Solvent A consisted of $97.8 \% \mathrm{H} 2 \mathrm{O}, 2 \%$ acetonitrile, 
414 and $0.2 \%$ formic acid and solvent $B$ consisted of $19.8 \% \mathrm{H} 2 \mathrm{O}, 80 \% \mathrm{ACN}$, and $0.2 \%$ formic 415 acid. The QExactive HF Orbitrap was operated in data dependent mode with the Tune

416 (version 2.7 SP1build 2659) instrument control software. Spray voltage was set to 2.5

$417 \mathrm{kV}$, S-lens RF level at 50, and heated capillary at $275^{\circ} \mathrm{C}$. Full scan resolution was set to

41860,000 at $\mathrm{m} / \mathrm{z} 200$. Full scan target was $3 \times 106$ with a maximum injection time of $15 \mathrm{~ms}$.

419 Mass range was set to $300-1650 \mathrm{~m} / \mathrm{z}$. For data dependent MS2 scans the loop count 420 was 12 , target value was set at $1 \times 105$, and intensity threshold was kept at $1 \times 105$.

421 Isolation width was set at $1.2 \mathrm{~m} / \mathrm{z}$ and a fixed first mass of 100 was used. Normalized 422 collision energy was set at 28. Peptide match was set to off, and isotope exclusion was 423 on. Data acquisition was controlled by Xcalibur (4.0.27.13) and all data was acquired in 424 profile mode.

425 Bioinformatics Putative BAR domain SO_1507 (BdpA) was identified in search of 426 annotation terms of $S$. oneidensis MR-1. The conserved domain database (CDD427 search)(NCBI) was accessed to identify the position-specific scoring matrix (PSSM) of 428 the specific region of SO_1507 that represented the BAR domain (amino acid residues 429 at positions 276-421). The domain prediction matched to BAR superfamily cl12013 and 430 specifically to the family member BAR cd07307. LOGICOIL multi-state coiled-coil 431 oligomeric state prediction was used to predict the presence of coiled-coils within BdpA

432 (43). SignalP 6.1 was used to detect the presence of the signal peptide and cellular 433 localization of BdpA (74). I-TASSER protein structure prediction (47) was used to 434 generate a predicted model of the BAR domain region of BdpA. Alignment of the predicted 435 BdpA BAR domain structure model to the structure of Hof1p was performed in the PyMOL 436 Molecular Graphics System, Version 2.0 (Schrödinger, LLC). 
A PSI-BLAST (75) search against the NCBI nr database was performed using the BdpA BAR sequence as the initial search seed to determine how prevalent the BdpA BAR domain is in related species. Conserved BdpA orthologs were annotated as hypothetical proteins in all of the species identified. In the initial round, 24 proteins were found from other organisms identified as Shewanella with a high conservation among the proteins and another 28 proteins were found in more distant bacteria species that had similarity of $65 \%$ to $44 \%$. A second iteration identified a few proteins much more distantly related from bacterial species and then proteins from eukaryote phylum Arthropoda that were annotated as being centrosomal proteins. All of the found proteins from bacterial species were hypothetical proteins with no known function. Only five of the proteins from the search returned hits to the PSSM of the BAR cd07307. The identity among the proteins was very high and examination of the proteins suggests that a functional form similar to 449 the BAR domain would result for all the found proteins. Overall, this places BdpA as a protein that just barely meets criteria via PSSM models to be assigned as matching the BAR domain while the rest of the proteins found have enough differences to fail to match

452 the BAR model while still being very similar to BdpA. An attempt was made to build up a HMM (Hidden Markov Model) using hmmer (76) to use for searching for other proteins

454 that might match, but as with the PSI-BLAST search, only the proteins that formed the 455 model returned as good matches. There appears to be a tight clade of very similar 456 proteins with very little differentiation in the sequence. This indicates that while sequence 457 homology between BdpA and the existing BAR domain consensus sequence predicted 458 the BAR domain region in BdpA using hmmer or $\mathrm{NCBl}$ tools, the sequence conservation 459 is at the cusp of a positive hit by the HMM since other closely related (>90\% homology) 
460 BdpA orthologs were not predicted to contain a BAR domain by this method. The most 461 homologous eukaryotic protein to BdpA $(27 \%)$ is a putative centrosomal protein in

462 Vollenhovia emeryi (accession \#: XP_011868153) that is predicted to contain an amino

463 terminal C2 membrane binding domain and a carboxy-terminal SMC domain within a

464 coiled-coil region. Despite CDD search failing to predict the presence of a BAR domain

465 in this protein, it does not preclude the presence of one, pending an updated BAR Pfam

466 HMM. Alignments and phylogenies were constructed in MEGA 7. MUSCLE was used to

467 align protein sequences, and Maximum Likelihood phylogenies were inferred using the 468 Le-Gascuel (LG+G) substitution matrix (77). Initial trees for the heuristic search were 469 obtained automatically by applying Neighbor-Join and BioNJ algorithms to a matrix of 470 pairwise distances estimated using a JTT model, and then selecting the topology with

471 superior log likelihood value. A discrete Gamma distribution was used to model

472 evolutionary rate differences among sites as indicated in figure legends. All positions with 473 less than $85 \%$ site coverage were eliminated.

474 Confocal microscopy For in vivo imaging of intrinsic outer membrane extension 475 production, S. oneidensis MR-1 strains were grown in LB media overnight, washed twice 476 with SDM, and diluted to an OD600 of 0.05 in $1 \mathrm{~mL}$ of SDM with appropriate antibiotics. 477 Prior to pipetting, $\sim 1 \mathrm{~cm}$ of the pipette tip was trimmed to minimize shear forces during 478 transfer. $100 \mu \mathrm{L}$ of each culture was labeled with $1 \mu \mathrm{L} 1 \mathrm{M}$ FM 4-64 to visualize the cell 479 membranes. After staining, $10 \mu \mathrm{L}$ of the labeled cell suspension was gently pipetted onto $48022 \times 22 \mathrm{~mm}$ No.1 cover glass (VWR) and sealed onto glass slides with clear acrylic nail 481 polish for confocal imaging or onto Lab-Tek chambered \#1 cover glass (Thermo Fischer 482 Scientific)(for widefield fluorescence). On average, intrinsic membrane extension 
483 formation could be observed starting after 45 minutes sealed onto cover glass. For 484 planktonic OME production from BdpA induction in trans, diluted cells (in either SDM for

485 S. oneidensis, BB for M. atlanticus, or LB for E. coli) were induced with $12.5 \mu \mathrm{M}$ DAPG 486 for 1 hour at $30^{\circ} \mathrm{C}$ with 200 RPM shaking agitation. Cells were labeled with FM 4-64 and 487 sealed onto glass slides as before. Induced OMEs were imaged immediately after 488 mounting onto slides.

Confocal images were taken by a Zeiss LSM 800 confocal microscope with a PlanApochromat 63x/1.4 numerical aperture oil immersion M27 objective. FM 4-64 fluorescence was excited at $506 \mathrm{~nm}$ : 0.20\% laser power. Emission spectra was detected 492 from 592-700 nm using the LSM 800 GaAsP-Pmt2 detector. To capture the dynamics of 493 the OMEs, images were collected over the designated length of time between $0.27-0.63$ 494 seconds per frame. Single frame time series images were collected of either a $50.71 \mu \mathrm{m}$ 495 by $50.71 \mu \mathrm{m}(2 \mathrm{x}$ zoom) or a $20.28 \mu \mathrm{m}$ by $20.28 \mu \mathrm{m}(5 \mathrm{x}$ zoom) field of view. Widefield fluorescence images were taken using a LED-Module $511 \mathrm{~nm}$ light source at $74.2 \%$ 497 intensity with 583-600 nm filters and a $91 \mathrm{He}$ CFP/YFP/mCherry reflector. Excitation and emission spectra were $506 \mathrm{~nm}$ and $751 \mathrm{~nm}$, respectively. Images were collected using a 499 Hamamatsu camera with a $250 \mathrm{~ms}$ exposure time. Images were recorded using the Zeiss 500 Zen software (Carl Zeiss Microscopy, LLC, Thornwood, NY, USA). Frequency of OMEs 501 was calculated from 3 biological replicates of each strain from at least 5 fields of view 502 selected at random (2444 cells from WT, 4378 from $\Delta b d p A$, and 3354 from $\Delta b d p A$ p452$503 \quad b d p A)$. OMEs were counted per cell per field of view from a 20 second video if a 504 membrane extension was observed extending from the cell over that timeframe. 505 Statistical significance was determined by two-tailed Student's $t$-test. 
Perfusion flow microscopy For OME statistics comparing S. oneidensis strains MR-1 Bertani (LB) broth (supplemented with $50 \mu \mathrm{g} / \mathrm{mL}$ Kanamycin for strains with plasmid) in a $125-\mathrm{mL}$ flask overnight at $30^{\circ} \mathrm{C}$ and $225 \mathrm{rpm}$. The next day, the stationary phase $\left(\mathrm{OD}_{600}\right.$ $\mathrm{mL}$ flask. After $\sim 6$ hours at $30^{\circ} \mathrm{C}$ and $225 \mathrm{rpm}$, when the $\mathrm{OD}_{600}$ was 2.4 (late log phase),

$5125 \mathrm{~mL}$ of cells were collected by centrifugation at $4226 \times \mathrm{g}$ for $5 \mathrm{~min}$ and washed twice in 513 defined medium. The perfusion chamber, microscope, and flow medium described previously (20-22) were used for all perfusion flow OME statistics experiments. During each 5 hour imaging experiment, the perfusion chamber was first filled with this flow 516 medium, then $<1 \mathrm{~mL}$ of washed cells were slowly injected for a surface density of $\sim 100$ -

517300 cells per $112 \times 112 \mu \mathrm{m}$ field of view on a Nikon Eclipse Ti-E inverted microscope with 518 the NIS-Elements AR software. Cells were allowed to attach for 5-15 minutes on the coverslip before perfusion flow was resumed at a volumetric flow rate of $6.25 \pm 0.1 \mu \mathrm{L} / \mathrm{s}$.

520 Cells and OMEs were visualized with the red membrane stain FM 4-64FX in the flow medium $(0.25 \mu \mathrm{g} / \mathrm{mL}$ of flow medium). A total of 1,831 wild type and 2,265 $\Delta b d p A$ cells

522 were used for extension and vesicle quantification. Experiments were performed from

523 three individual biological replicates for each strain, and statistical significance was 524 determined by two-tailed $x^{2}$ test.

\section{Cryo transmission electron microscopy}

526 Shewanella strains were streaked onto LB plates with or without kanamycin and allowed

527 to incubate 3 days on a benchtop. The night before freezing, individual colonies were 528 inoculated into $3 \mathrm{ml} \mathrm{LB}+/$ - kanamycin and incubated at $30{ }^{\circ} \mathrm{C}$ overnight with $200 \mathrm{rpm}$ 
529 shaking. The following morning optical densities of the cultures were measured at

$530600 \mathrm{~nm}$ and adjusted to a final OD 600 of 1 . Cells were pelleted at $8,000 \mathrm{rpm}$ for three

531 minutes for buffer exchange/washes. For the $\Delta b d p A$ p452-bdpA transformed cells, 12.5

$532 \mu \mathrm{M}$ DAPG was added. A freshly glow discharged 200 mesh copper grid with R2/1

533 Quantifoil carbon film was placed into a concavity slide. Approximately $150 \mu$ of a 1:10

534 dilution of the cell suspensions, with or without the inducer, was added to cover the grid.

535 A glass coverslip was then lowered onto the concavity to exclude air bubbles. The

536 edges of the coverslip were then sealed with nail polish to prevent media evaporation.

537 The slide assembly was then incubated in a $30{ }^{\circ} \mathrm{C}$ incubator for 1.5 to 3 hours.

538 Immediately prior to plunge freezing, the top coverslip was removed by scoring the nail

539 polish with a razor blade. TEM grids with cells were gently retrieved with forceps and

540 loaded into a Leica grid plunge for automated blotting and plunging into LN2-cooled

541 liquid ethane. Vitrified grids were transferred to a $L N_{2}$ storage dewar. Imaging of frozen

542 samples was performed on either a Titan (ThermoFisher Scientific) microscope

543 equipped with a Gatan Ultrascan camera and operating at $300 \mathrm{kV}$ or a Talos

544 (ThermoFisher Scientific) equipped with a Ceta camera and operating at $200 \mathrm{kV}$.

545 Images were acquired at 10,000x to 20,000x magnification and were adjusted by

546 bandpass filtering. Unfixed OMEs were sorted based on appearance into 4 categories.

547 Tubules were narrow OMEs with relatively uniform or slight symmetric curvature.

548 Narrow chains were recorded as OMEs with a narrow, consistent diameter and

549 symmetric curvature at constriction points. Irregular chains were classified as OMEs

550 without a consistent diameter throughout the length of the OME and asymmetric

551 curvature on either side of the extension. Blebs \bulges were outer membrane 
552 structures that did not resemble OMEs but still extended from the cell membrane

553 surface. Phenotypes were documented from observations of $31 \mathrm{WT}, 13 \Delta b d p A$, and 3

$554 \triangle b d p A$ p452-bdpA OMEs over three separate biological replicates, with two technical

555 replicates of each strain per biological replicate. Two-tailed statistical significance

556 between strains was calculated by Fisher's exact test with the Freeman-Halton

557 extension for a 2x4 matrix (78) using the Real Statistics Resource Pack software

558 (Release 6.8).

\section{Acknowledgements}

We thank Dr. Jeffery Gralnick for helpful discussions and advice; Dr. Adam Meyer

562 and Dr. Chris Voigt for the DAPG-inducible Marionette promoter; Dr. Annie Moradian and

Dr. Mike Sweredoski and the California Institute of Technology Proteome Exploration Lab

564 for useful discussions on the preparation and analysis of proteomics data. Some of the cryo-TEM work was done in the Beckman Institute Resource Center for Transmission

566 Electron Microscopy at Caltech. This work was supported by the United States

567 Department of Defense Synthetic Biology for Military Environments (SBME) Applied

568 Research for the Advancement of Science and Technology Priorities (ARAP) program.

569 Work in ME-N's lab was supported by the U.S. Office of Naval Research Multidisciplinary

570 University Research Initiative Grant No. N00014-18-1-2632. LAZ was partially supported

571 by the National Science Foundation grant DEB-1542527. SX was supported by the

572 Division of Chemical Sciences, Geosciences, and Biosciences, Office of Basic Energy

573 Sciences of the U.S. Department of Energy through grant DE-FG02-13ER16415. Work

574 in GJJ's lab was supported by the National Institute of Health (GM122588 to GJJ). 


\section{Author Contributions}

576 DP and LZ conceived the study independently then combined projects when

577 complementary data on BdpA was discovered. LZ purified OMVs, prepared samples for

578 LC MS-MS, and performed DLS measurements. LZ and SX made electrochemical

579 measurements and analysis. DP conducted BdpA domain prediction and validation 580 analysis, generated the p452-bdpA plasmid, $\Delta b d p A$ and $p 452-b d p A$ strains. DP and GC

581 conducted fluorescence imaging experiments, and DP, LZ, and GC analyzed the data.

582 LB adapted the Marionette sensor (P phlF-YFP) into pBBR1-mcs2. LZ and LAM performed

583 cryo-TEM of OMVs. GC and LZ analyzed perfusion flow system data. CH, DP, and LD

584 performed cryo-TEM experiments of OMEs and image processing / analysis. DP and AM 585 generated phylogenetic data, and DP, AM, and BE analyzed the data. DP, LZ, CM, GC,

586 AM, LAM, BE, GJJ, LD, MEN, and SG provided data interpretation. DP, LZ, MEN, and 587 SG wrote the manuscript, with input from all coauthors. 


\section{References}

590 1. Peter BJ, et al. (2004) BAR domains as sensors of membrane curvature: the amphiphysin BAR structure. Science 303(5657):495-499.

2. Frost A, De Camilli P, \& Unger VM (2007) F-BAR proteins join the BAR family fold. Structure 15(7):751-753.

3. Shimada A, et al. (2007) Curved EFC/F-BAR-domain dimers are joined end to end into a filament for membrane invagination in endocytosis. Cell 129(4):761-772.

4. Weissenhorn W (2005) Crystal structure of the endophilin-A1 BAR domain. J. Mol. Biol. 351(3):653-661.

5. Drin G \& Antonny B (2010) Amphipathic helices and membrane curvature. FEBS Lett. 584(9):1840-1847.

6. Seet LF \& Hong W (2006) The Phox (PX) domain proteins and membrane traffic. Biochim. Biophys. Acta 1761(8):878-896.

7. Itoh T \& De Camilli P (2006) BAR, F-BAR (EFC) and ENTH/ANTH domains in the regulation of membrane-cytosol interfaces and membrane curvature. Biochim. Biophys. Acta 1761(8):897912.

8. Knaevelsrud $\mathrm{H}$, et al. (2013) Membrane remodeling by the PX-BAR protein SNX18 promotes autophagosome formation. J. Cell Biol. 202(2):331-349.

9. Simunovic M, Voth GA, Callan-Jones A, \& Bassereau P (2015) When Physics Takes Over: BAR Proteins and Membrane Curvature. Trends Cell Biol. 25(12):780-792.

10. Mim C, et al. (2012) Structural basis of membrane bending by the N-BAR protein endophilin. Cell 149(1):137-145.

11. Habermann B (2004) The BAR-domain family of proteins: a case of bending and binding? EMBO reports 5(3):250-255.

12. Miki H, Yamaguchi H, Suetsugu S, \& Takenawa T (2000) IRSp53 is an essential intermediate between Rac and WAVE in the regulation of membrane ruffling. Nature 408(6813):732-735.

13. Carman PJ \& Dominguez R (2018) BAR domain proteins-a linkage between cellular membranes, signaling pathways, and the actin cytoskeleton. Biophys Rev 10(6):1587-1604.

14. Toyofuku M, Nomura N, \& Eberl L (2018) Types and origins of bacterial membrane vesicles. Nature Reviews Microbiology.

15. Remis JP, et al. (2014) Bacterial social networks: structure and composition of Myxococcus xanthus outer membrane vesicle chains. Environ. Microbiol. 16(2):598-610.

16. Wei X, Vassallo CN, Pathak DT, \& Wall D (2014) Myxobacteria produce outer membraneenclosed tubes in unstructured environments. J. Bacteriol. 196(10):1807-1814.

17. Fischer T, et al. (2019) Biopearling of Interconnected Outer Membrane Vesicle Chains by a Marine Flavobacterium. Appl. Environ. Microbiol. 85(19).

18. Hampton CM, et al. (2017) The Opportunistic Pathogen Vibrio vulnificus Produces Outer Membrane Vesicles in a Spatially Distinct Manner Related to Capsular Polysaccharide. Front. Microbiol. 8:2177.

19. Sampath V, McCaig WD, \& Thanassi DG (2018) Amino acid deprivation and central carbon metabolism regulate the production of outer membrane vesicles and tubes by Francisella. Mol. Microbiol. 107(4):523-541. 
20. Chong GW, Pirbadian S, \& El-Naggar MY (2019) Surface-Induced Formation and RedoxDependent Staining of Outer Membrane Extensions in Shewanella oneidensis MR-1. Front. Energy Res. 7.

21. Subramanian P, Pirbadian S, El-Naggar MY, \& Jensen GJ (2018) Ultrastructure of Shewanella oneidensis MR-1 nanowires revealed by electron cryotomography. Proc. Natl. Acad. Sci. U. S. A. 115(14):E3246-E3255.

22. Pirbadian S, et al. (2014) Shewanella oneidensis MR-1 nanowires are outer membrane and periplasmic extensions of the extracellular electron transport components. Proc. Natl. Acad. Sci. U. S. A. 111(35):12883-12888.

23. Gorby YA, et al. (2006) Electrically conductive bacterial nanowires produced by Shewanella oneidensis strain MR-1 and other microorganisms. Proc. Natl. Acad. Sci. U. S. A. 103(30):1135811363.

24. Bhattacharya S, et al. (2019) A Ubiquitous Platform for Bacterial Nanotube Biogenesis. Cell Rep.

25. Dubey GP, et al. (2016) Architecture and Characteristics of Bacterial Nanotubes. Dev. Cell 36(4):453-461.

26. Dubey GP \& Ben-Yehuda S (2011) Intercellular nanotubes mediate bacterial communication. Cell 144(4):590-600.

27. Pande $\mathrm{S}$, et al. (2015) Metabolic cross-feeding via intercellular nanotubes among bacteria. Nat Commun 6:6238.

28. Tanaka M, Arakaki A, \& Matsunaga T (2010) Identification and functional characterization of liposome tubulation protein from magnetotactic bacteria. Mol. Microbiol. 76(2):480-488.

29. Danne L, et al. (2017) Membrane Remodeling by a Bacterial Phospholipid-Methylating Enzyme. MBio 8(1).

30. Danne L, et al. (2017) Dissection of membrane-binding and-remodeling regions in two classes of bacterial phospholipid N-methyltransferases. Biochim. Biophys. Acta 1859(12):2279-2288.

31. Danne L, et al. (2015) Membrane-binding mechanism of a bacterial phospholipid Nmethyltransferase. Mol. Microbiol. 95(2):313-331.

32. Low HH, Sachse C, Amos LA, \& Lowe J (2009) Structure of a bacterial dynamin-like protein lipid tube provides a mechanism for assembly and membrane curving. Cell 139(7):1342-1352.

33. Low HH \& Lowe J (2006) A bacterial dynamin-like protein. Nature 444(7120):766-769.

34. Bohuszewicz O, Liu J, \& Low HH (2016) Membrane remodelling in bacteria. J. Struct. Biol. 196(1):3-14.

35. Nealson KH \& Scott J (2006) Ecophysiology of the Genus Shewanella.1133-1151.

36. Marsili E, et al. (2008) Shewanella secretes flavins that mediate extracellular electron transfer. Proceedings of the National Academy of Sciences 105(10):3968-3973.

37. Gorby Y, et al. (2008) Redox-reactive membrane vesicles produced by Shewanella. Geobiology 6(3):232-241.

38. Liu X, et al. (2019) Bacterial Vesicles Mediate Extracellular Electron Transfer. Environmental Science \& Technology Letters.

39. Xu S, Barrozo A, Tender LM, Krylov Al, \& El-Naggar MY (2018) Multiheme Cytochrome Mediated Redox Conduction through Shewanella oneidensis MR-1 Cells. J. Am. Chem. Soc. 140(32):1008510089.

40. Okamoto A, Nakamura R, \& Hashimoto K (2011) In-vivo identification of direct electron transfer from Shewanella oneidensis MR-1 to electrodes via outer-membrane OmcA-MtrCAB protein complexes. Electrochim. Acta 56(16):5526-5531.

41. Cox J, et al. (2014) Accurate proteome-wide label-free quantification by delayed normalization and maximal peptide ratio extraction, termed MaxLFQ. Mol. Cell. Proteomics 13(9):2513-2526. 
678 42. Hsu VW, Bai M, \& Li J (2012) Getting active: protein sorting in endocytic recycling. Nat. Rev. Mol. Cell Biol. 13(5):323-328.

43. Vincent TL, Green PJ, \& Woolfson DN (2013) LOGICOIL--multi-state prediction of coiled-coil oligomeric state. Bioinformatics 29(1):69-76.

44. Linkner J, et al. (2014) The inverse BAR domain protein IBARa drives membrane remodeling to control osmoregulation, phagocytosis and cytokinesis. J. Cell Sci. 127(Pt 6):1279-1292.

45. Cui $\mathrm{H}$, et al. (2013) Understanding the role of amphipathic helices in N-BAR domain driven membrane remodeling. Biophys. J. 104(2):404-411.

46. Henne WM, et al. (2007) Structure and analysis of FCHo2 F-BAR domain: a dimerizing and membrane recruitment module that effects membrane curvature. Structure 15(7):839-852.

47. Roy A, Kucukural A, \& Zhang Y (2010) I-TASSER: a unified platform for automated protein structure and function prediction. Nat. Protoc. 5(4):725-738.

48. Moravcevic K, et al. (2015) Comparison of Saccharomyces cerevisiae F-BAR domain structures reveals a conserved inositol phosphate binding site. Structure 23(2):352-363.

49. van Weering JR \& Cullen PJ (2014) Membrane-associated cargo recycling by tubule-based endosomal sorting. Semin. Cell Dev. Biol. 31:40-47.

50. Pylypenko O, Lundmark R, Rasmuson E, Carlsson SR, \& Rak A (2007) The PX-BAR membraneremodeling unit of sorting nexin 9. EMBO J. 26(22):4788-4800.

51. Vinogradov E, Korenevsky A, \& Beveridge TJ (2003) The structure of the rough-type lipopolysaccharide from Shewanella oneidensis MR-1, containing 8-amino-8-deoxy-Kdo and an open-chain form of 2-acetamido-2-deoxy-d-galactose. Carbohydr. Res. 338(19):1991-1997.

52. Daumke O, Roux A, \& Haucke V (2014) BAR domain scaffolds in dynamin-mediated membrane fission. Cell 156(5):882-892.

53. Schoneberg J, et al. (2017) Lipid-mediated PX-BAR domain recruitment couples local membrane constriction to endocytic vesicle fission. Nat Commun 8:15873.

54. Pinheiro PS, et al. (2014) The BAR domain protein PICK1 controls vesicle number and size in adrenal chromaffin cells. J. Neurosci. 34(32):10688-10700.

55. Meyer AJ, Segall-Shapiro TH, Glassey E, Zhang J, \& Voigt CA (2018) Escherichia coli "Marionette" strains with 12 highly optimized small-molecule sensors. Nat. Chem. Biol.

56. Mahmood MI, Noguchi H, \& Okazaki KI (2019) Curvature induction and sensing of the F-BAR protein Pacsin1 on lipid membranes via molecular dynamics simulations. Sci. Rep. 9(1):14557.

57. Wang $Q$, et al. (2009) Molecular mechanism of membrane constriction and tubulation mediated by the F-BAR protein Pacsin/Syndapin. Proc. Natl. Acad. Sci. U. S. A. 106(31):12700-12705.

58. Bird L, et al. (2018) Development of a Genetic System for Marinobacter atlanticus CP1 (sp. nov.), a Wax Ester Producing Strain Isolated From an Autotrophic Biocathode. Front. Microbiol. 9:3176.

59. Coursolle D \& Gralnick JA (2010) Modularity of the Mtr respiratory pathway of Shewanella oneidensis strain MR-1. Mol. Microbiol. 77(4):995-1008.

60. Bird LJ, et al. (2019) Engineered living conductive biofilms as functional materials. MRS Commun.:1-13.

61. Ayton GS, et al. (2009) New insights into BAR domain-induced membrane remodeling. Biophys. J. 97(6):1616-1625.

62. Simunovic M, et al. (2013) Protein-mediated transformation of lipid vesicles into tubular networks. Biophys. J. 105(3):711-719.

63. Noguchi H (2016) Membrane tubule formation by banana-shaped proteins with or without transient network structure. Sci. Rep. 6:20935.

64. Stachowiak JC, et al. (2012) Membrane bending by protein-protein crowding. Nat. Cell Biol. 14(9):944-949. 
65. Frohlich C, et al. (2013) Structural insights into oligomerization and mitochondrial remodelling of dynamin 1-like protein. EMBO J. 32(9):1280-1292.

66. Ford MG, et al. (2002) Curvature of clathrin-coated pits driven by epsin. Nature 419(6905):361366.

67. Yoon Y, et al. (2010) Molecular basis of the potent membrane-remodeling activity of the epsin 1 N-terminal homology domain. J. Biol. Chem. 285(1):531-540.

68. Salzer U, Kostan J, \& Djinovic-Carugo K (2017) Deciphering the BAR code of membrane modulators. Cell. Mol. Life Sci. 74(13):2413-2438.

69. Perez-Cruz C, et al. (2013) New type of outer membrane vesicle produced by the Gram-negative bacterium Shewanella vesiculosa M7T: implications for DNA content. Appl. Environ. Microbiol. 79(6):1874-1881.

70. Hagen WJH, Wan W, \& Briggs JAG (2017) Implementation of a cryo-electron tomography tiltscheme optimized for high resolution subtomogram averaging. J. Struct. Biol. 197(2):191-198.

71. Rohou A \& Grigorieff N (2015) CTFFIND4: Fast and accurate defocus estimation from electron micrographs. J. Struct. Biol. 192(2):216-221.

72. Kremer JR, Mastronarde DN, \& McIntosh JR (1996) Computer visualization of three-dimensional image data using IMOD. J. Struct. Biol. 116(1):71-76.

73. Brown RN, Romine MF, Schepmoes AA, Smith RD, \& Lipton MS (2010) Mapping the subcellular proteome of Shewanella oneidensis MR-1 using sarkosyl-based fractionation and LC-MS/MS protein identification. J. Proteome Res. 9(9):4454-4463.

74. Nielsen H (2017) Predicting Secretory Proteins with SignalP. Protein Function Prediction: Methods and Protocols, ed Kihara D (Springer New York, New York, NY), pp 59-73.

75. Altschul SF, et al. (1997) Gapped BLAST and PSI-BLAST: a new generation of protein database search programs. Nucleic Acids Res. 25(17):3389-3402.

76. Finn RD, Clements J, \& Eddy SR (2011) HMMER web server: interactive sequence similarity searching. Nucleic Acids Res. 39(Web Server issue):W29-37.

77. Le SQ \& Gascuel O (2008) An improved general amino acid replacement matrix. Mol. Biol. Evol. 25(7):1307-1320.

78. Freeman GH \& Halton JH (1951) Note on an exact treatment of contingency, goodness of fit and other problems of significance. Biometrika 38(1-2):141-149.

79. Myers CR \& Nealson KH (1988) Bacterial manganese reduction and growth with manganese oxide as the sole electron acceptor. Science 240(4857):1319-1321.

80. Saltikov CW \& Newman DK (2003) Genetic identification of a respiratory arsenate reductase. Proc. Natl. Acad. Sci. U. S. A. 100(19):10983-10988.

81. Kovach ME, et al. (1995) Four new derivatives of the broad-host-range cloning vector pBBR1MCS, carrying different antibiotic-resistance cassettes. Gene 166(1):175-176.

82. Simon R, Priefer U, \& Pühler A (1983) A Broad Host Range Mobilization System for In Vivo Genetic Engineering: Transposon Mutagenesis in Gram Negative Bacteria. Biotechnology. (N. Y.) 1(9):784-791. 

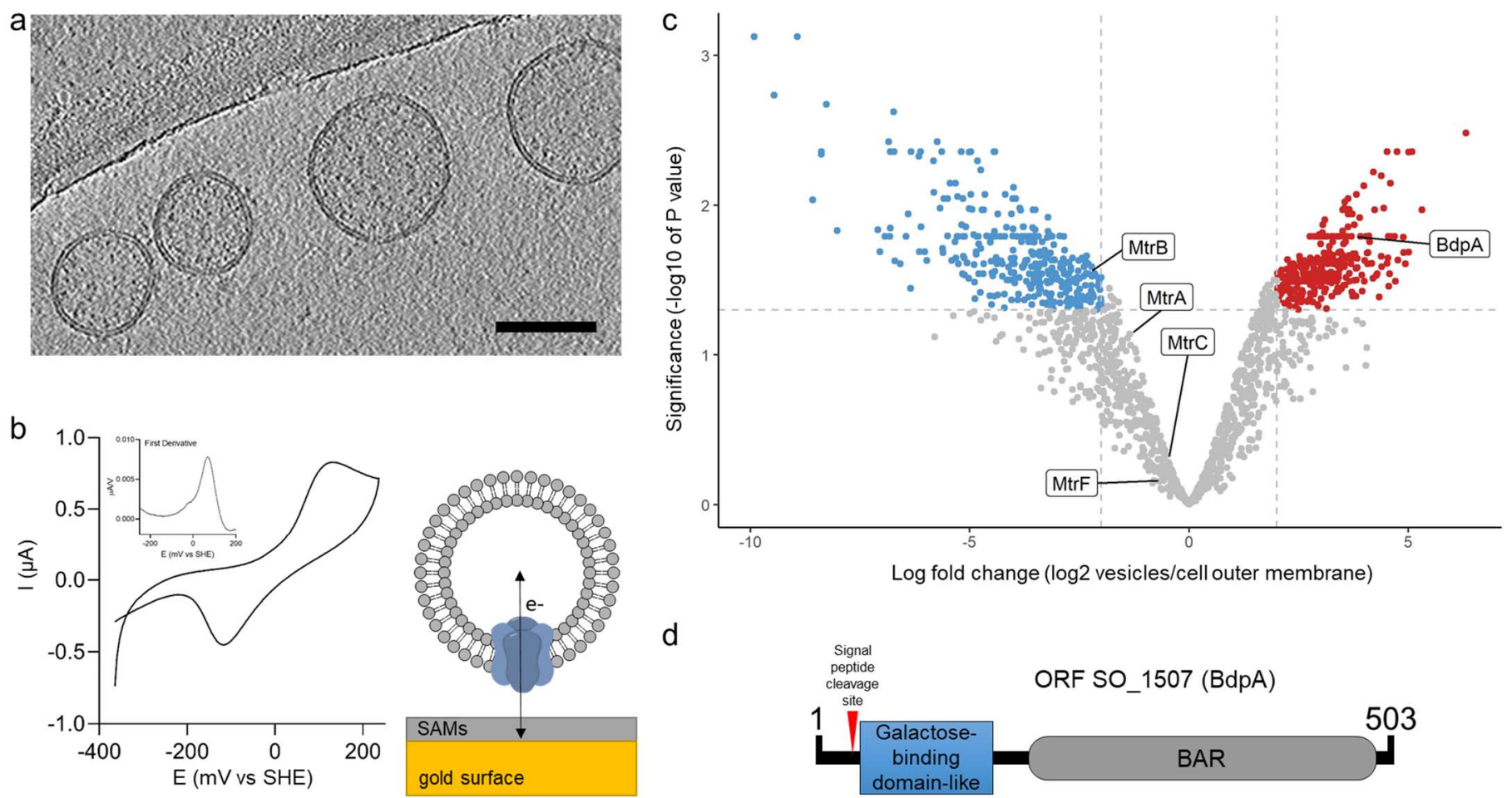

$d$

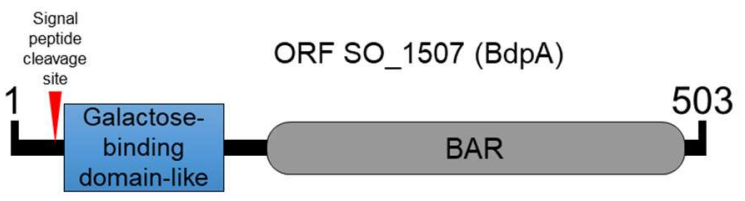

771 Fig. 1| Redox active vesicles are enriched with BAR domain protein BdpA. a.

772 Cryoelectron tomography image of $S$. oneidensis MR-1 outer membrane vesicles (OMVs)

773 (scale $=200 \mathrm{~nm}$ ). b. Cyclic voltammetry of vesicles adhered to gold electrode via small

774 self-assembled monolayers, as diagramed. Inset shows first derivative of anodic scan. c.

775 Volcano plot of vesicle proteome compared to cell-associated outer membrane (red = 776 enriched in vesicles, blue = enriched in cell-associated outer membrane). d. Schematic 777 of BdpA domains. 

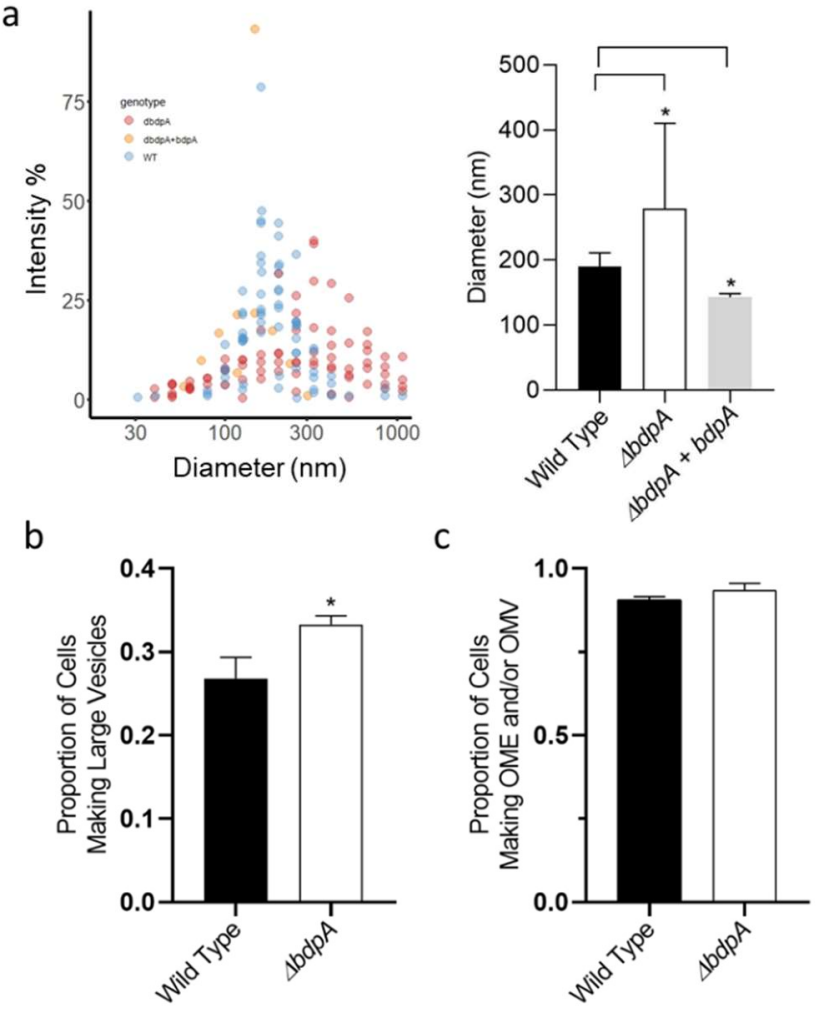

Fig. 2| BdpA is responsible for maintaining vesicle size but does not alter the frequency of OMV or OME formation. a. Dynamic light scattering of OMV size distributions from the deletion strain (red, 9 biological replicates) compared to wild type (blue, 11 biological replicates) and $\Delta b d p A$ cells expressing $b d p A$ from a plasmid (yellow, (left), with weighted averages of OMV diameters (right). Color opacity denotes overlapping data points ( $p<0.05, t$-test). b. Proportion of cells forming large vesicles (>300 nm diameter) during perfusion flow monitored by time lapse fluorescence imaging $\left(p<0.00459, x^{2}\right.$ test). c. Proportion of cells forming vesicles and extensions relative to the total number of cells observed by fluorescence microscopy during perfusion flow ( $p>$ $0.05, x^{2}$ test). Time lapse images were recorded from 3 biological replicates per strain with 5-13 fields of view per replicate. Error bars represent standard deviation. 

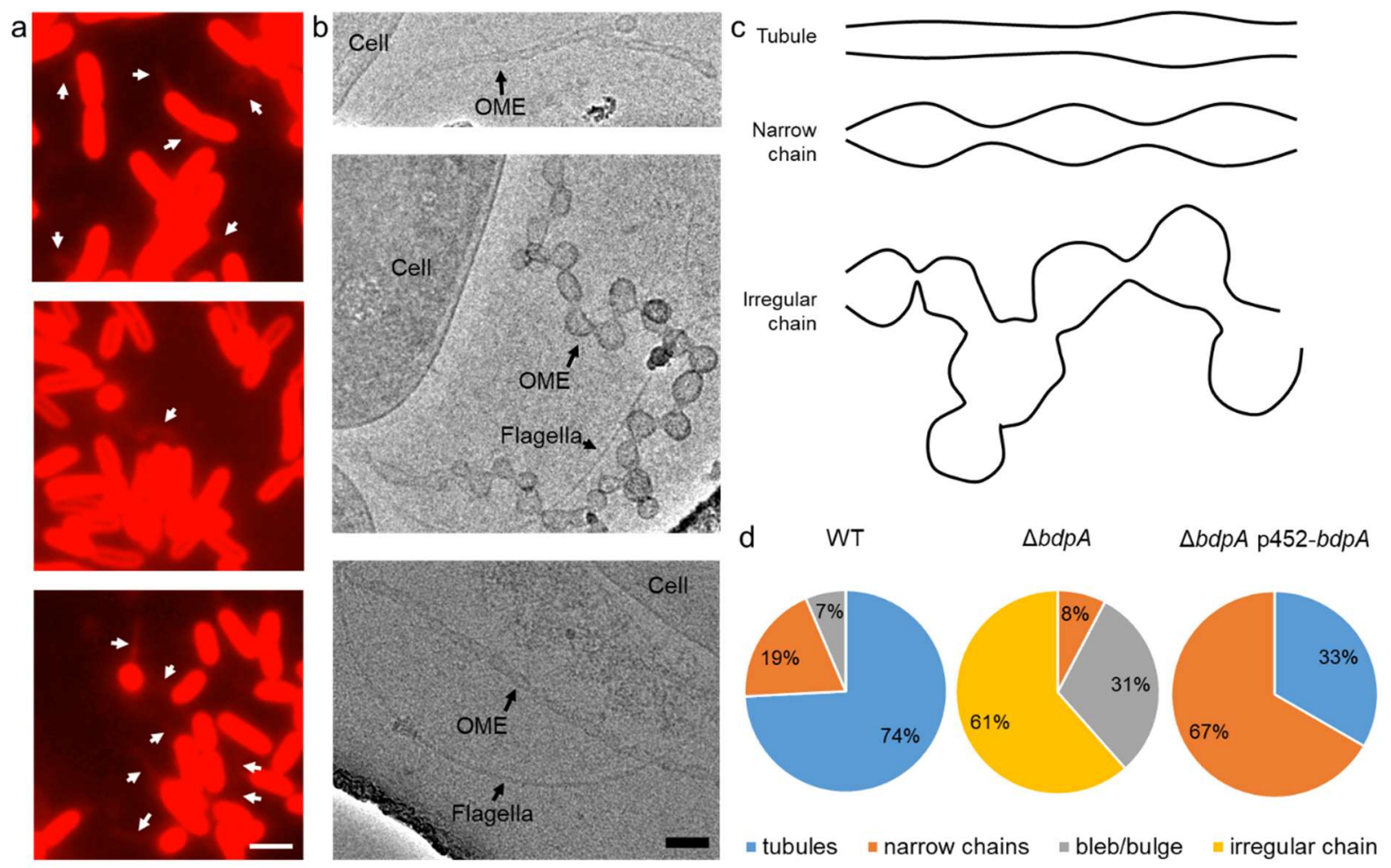

Fig. 3| BdpA promotes OME maturation into ordered tubules. a. Fluorescence images of $S$. oneidensis WT (top), $\triangle b d p A$ (middle), and $\triangle b d p A$ p452-bdpA with $12.5 \mu \mathrm{M}$ DAPG (bottom) OMEs. Scale $=2 \mu \mathrm{m}$. b. Cryo-TEM images of WT (top), $\triangle b d p A$ (middle), and $\triangle b d p A$ p452-bdpA with $12.5 \mu \mathrm{M}$ DAPG (bottom) OMEs. Scale $=100 \mathrm{~nm}$. c. Representative cartoon of OME phenotypes. d. Pie charts show relative frequency of OME phenotypes observed from each strain. Membrane blebs/bulges were defined as non-structured membrane protrusions that did not resemble either of the other OME categories. 

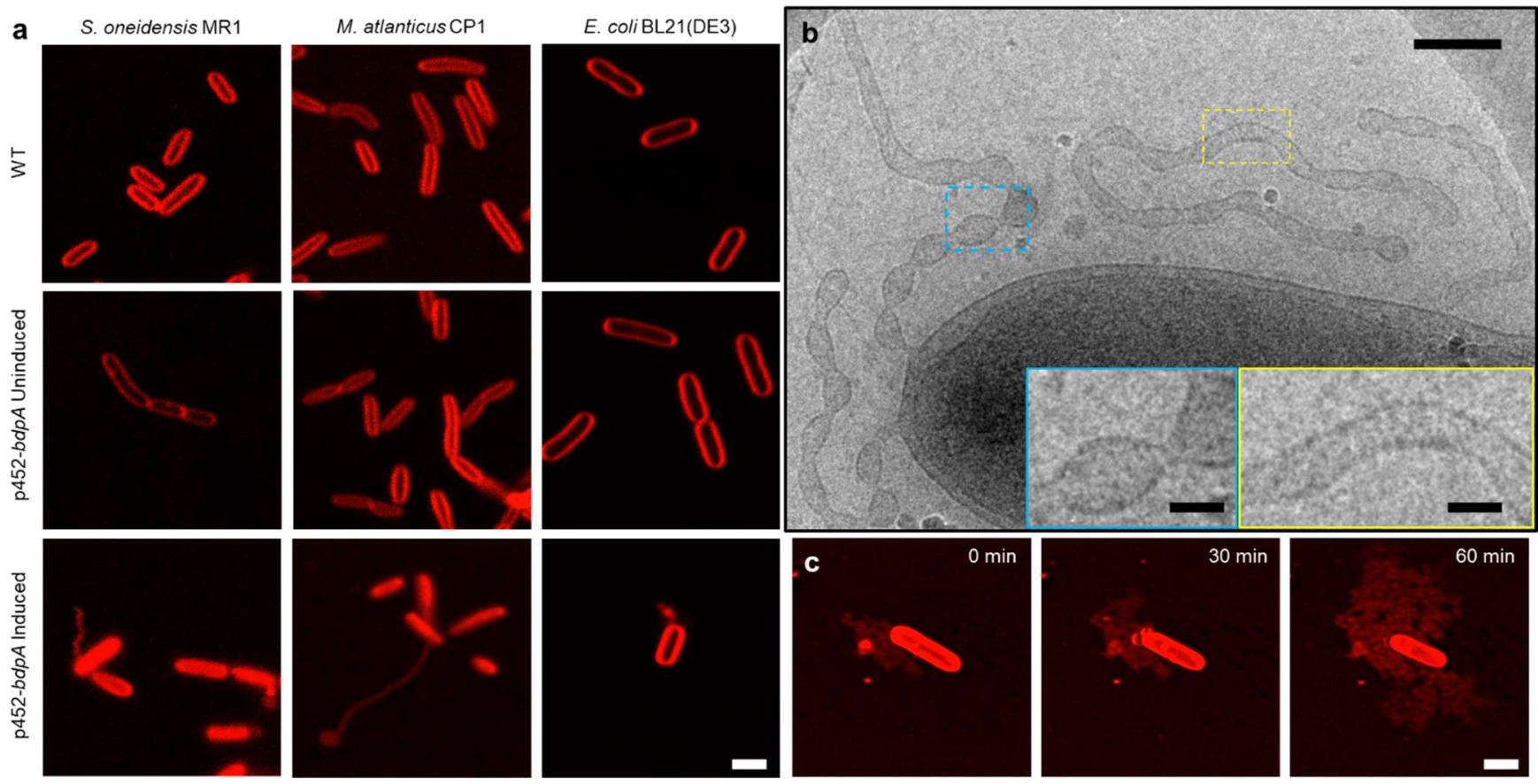

\section{4}

Fig. 4| Heterologous expression of BdpA promotes OME formation. a. Induction of BdpA expression with $12.5 \mu \mathrm{M}$ DAPG during planktonic, non-attached growth results in OME formation in S. oneidensis (left), M. atlanticus CP1 (middle), and E. coli BL21(DE3) (right). Scale $=2 \mu \mathrm{m}$. b. Cryo-TEM image of OMEs following planktonic induction of BdpA expression in S. oneidensis WT p452-bdpA cells. Scale $=200 \mathrm{~nm}$. Insets enlarged to show detail of regularly ordered electron densities at the surface of OME junctions (blue) and tubule regions (yellow). Scale $=50 \mathrm{~nm}$. c. OME growth over time at 30 minute intervals of $E$. coli BL21(DE3) expressing BdpA while attached to a glass surface. Scale $812=2 \mu \mathrm{m}$. 


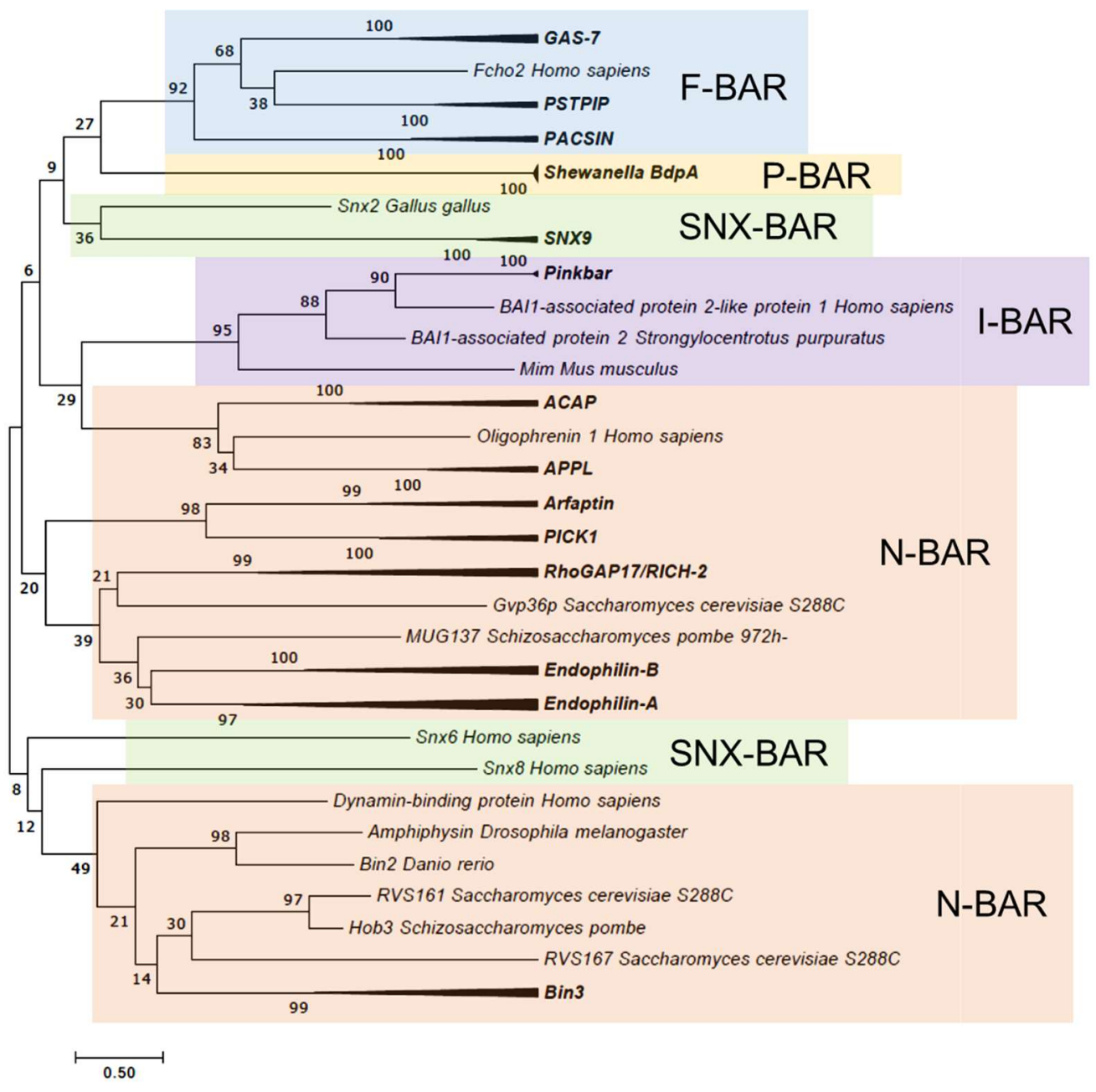

Fig. 5| Comparative phylogenetic analysis of BdpA with prokaryotic homologs and eukaryotic BAR domains. Maximum Likelihood evolutionary histories were inferred from 1000 bootstrap replicates, and the percentage of trees in which the taxa clustered together is shown next to the branches. Arrows indicate multiple branches collapsed to a single node. S. oneidensis BdpA and 5 prokaryotic orthologs (WP_011623497 unclassified Shewanella, ESE40074 - S. decolorationis S12, WP_039978560 - S. decolorationis, KEK29176 - S. xiamenensis, and WP_055648003 - Shewanella sp. Sh95) predicted by the current BAR domain Pfam HMM to contain a BAR domain aligned with representative BAR domains from various BAR domain subtypes (N-BAR, F-BAR, SNX-BAR, I-BAR) at a total of 196 positions. The Gamma distribution used to model evolutionary rate differences among sites was 12.9598. 


\section{Supplemental Table 2}

829

830

\begin{tabular}{|c|c|c|}
\hline Strain, Plasmid, or Primer & Description or relevant genotype & $\begin{array}{l}\text { Source or } \\
\text { reference }\end{array}$ \\
\hline \multicolumn{3}{|l|}{$\begin{array}{l}\text { Shewanella oneidensis } \\
\text { strains }\end{array}$} \\
\hline MR-1 & Wild type & {$[(79)]$} \\
\hline$\Delta b d p A$ & bdpA scarless deletion & This study \\
\hline $\begin{array}{l}\text { Marinobacter atlanticus CP1 } \\
\text { Eschericia coli strains }\end{array}$ & Wild type & {$[(58)]$} \\
\hline BL21(DE3) & OneShot E. coli BL21(DE3) & Invitrogen \\
\hline UQ950 & Cloning strain & {$[(80)]$} \\
\hline BW29427 (WM3064) & Conjugation strain & {$[(80)]$} \\
\hline \multicolumn{3}{|l|}{ Plasmids } \\
\hline pBBR1-mcs2 & empty vector & {$[(81)]$} \\
\hline pBBJM & Cloning backbone & This study \\
\hline pSMV3 & Suicide vector & {$[(82)]$} \\
\hline pSMV3_1507KO & $\begin{array}{l}\text { Contains up and downstream regions of } \\
\text { SO_1507 }\end{array}$ & This study \\
\hline $\mathrm{p} 452-b d p A$ & $\mathrm{DA} \overline{\mathrm{P}} \mathrm{G}$ inducible $b d p A$ vector & This study \\
\hline \multicolumn{3}{|l|}{ Primers } \\
\hline pAJMF2 & $\begin{array}{l}\text { TTAACGCGAATTTTAACAAAATATTAACGCcccgc } \\
\text { ttaacgatcgttggctg }\end{array}$ & \\
\hline pAJMR3 & $\begin{array}{l}\text { AGCGGATAACAATTTCACACAGGAAACAGCTac } \\
\text { ctcagataaaatatttgc }\end{array}$ & \\
\hline pBBRF3 & $\begin{array}{l}\text { gggctcatgagcaaatattttatctgaggtAGCTGTTTCCT } \\
\text { GTGTGAAATTG }\end{array}$ & \\
\hline pBBRR2 & $\begin{array}{l}\text { acccgcgctcagccaacgatcgttaagcgggGCGTTAATA } \\
\text { TTTTGTTAAAATTCGC }\end{array}$ & \\
\hline 1507F_insert & $\begin{array}{l}\text { ttaatactagagaaagaggggaaatactagATGCGCACC } \\
\text { GCTGC }\end{array}$ & \\
\hline 1507R_insert & $\begin{array}{l}\text { gaggcctcttttctggaatttggtaccgagCTACATAAAG } \\
\text { GCTTTAGTAAAGGCTT }\end{array}$ & \\
\hline BBJMV_reverse & $\begin{array}{l}\text { CAGCATTGAGATGACTGCAGCGGTGCGCATcta } \\
\text { gtatttcccctctttctctagtat }\end{array}$ & \\
\hline BBJMV_forward & $\begin{array}{l}\text { AAGGAAGCCTTTACTAAAGCCTTTATGTAGctcg } \\
\text { gtaccaaattccagaaaag }\end{array}$ & \\
\hline
\end{tabular}




\section{Supplemental Figure 1}

A

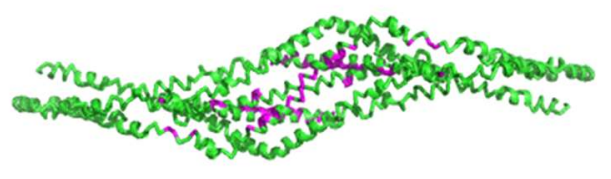

Top

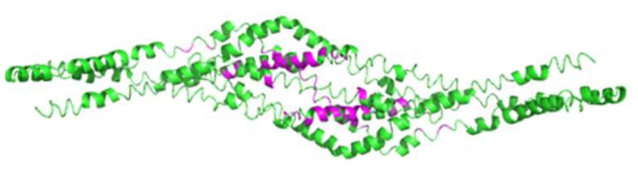

Bottom

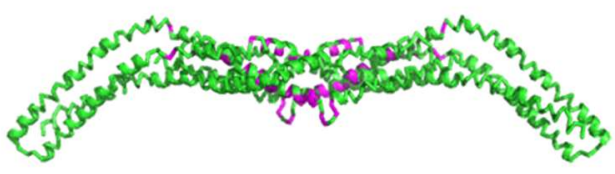

Side

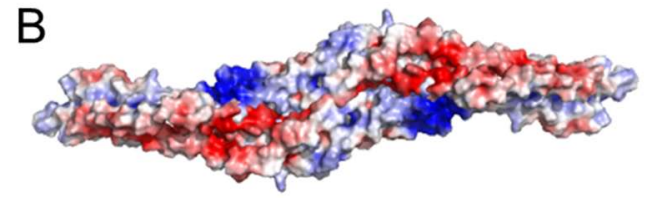

Top

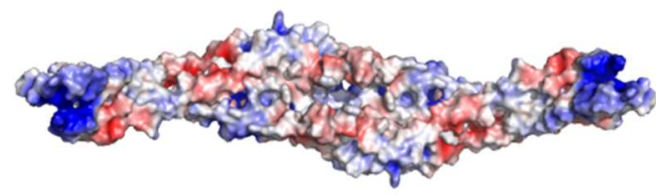

Bottom

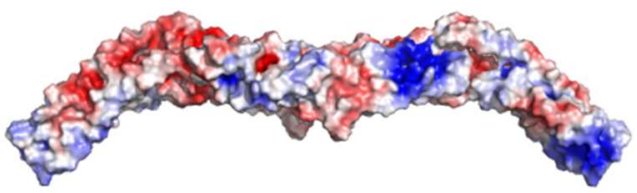

Side

834

Predicted structure of the BAR domain region of BdpA.

(A) Ribbon diagram of i-TASSER prediction of BdpA structure as a dimeric molecule, residues 175502. Dimer was modeled from an alignment of BdpA monomers to homodimeric Hof1p (Protein Data Bank [PDB] ID code 4WPE) structures that resulted in closest proximity of putative dimer interface (purple) residues from the initial BAR domain prediction. (B) Surface representation of predicted BdpA homodimer colored according to electrostatic potential. The bottom concave face has an accumulation of distributed positively charged residues (Lys, Arg, His - blue), while the top face has clusters of positive and negatively charged (Glu, Asp - red) residues, within a range of -3.0 to $3.0 \mathrm{~V}$. 


\section{Supplemental Figure 2}

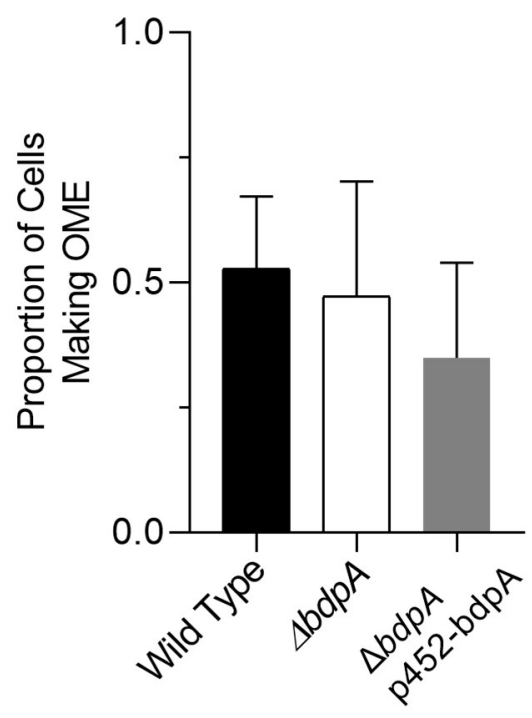

838 Proportion of cells making OMEs without perfusion flow at three hours post deposition onto chambered cover glass, measured from five random fields of view from each of three independent cultures per strain. 


\section{Supplemental Figure 3}

843
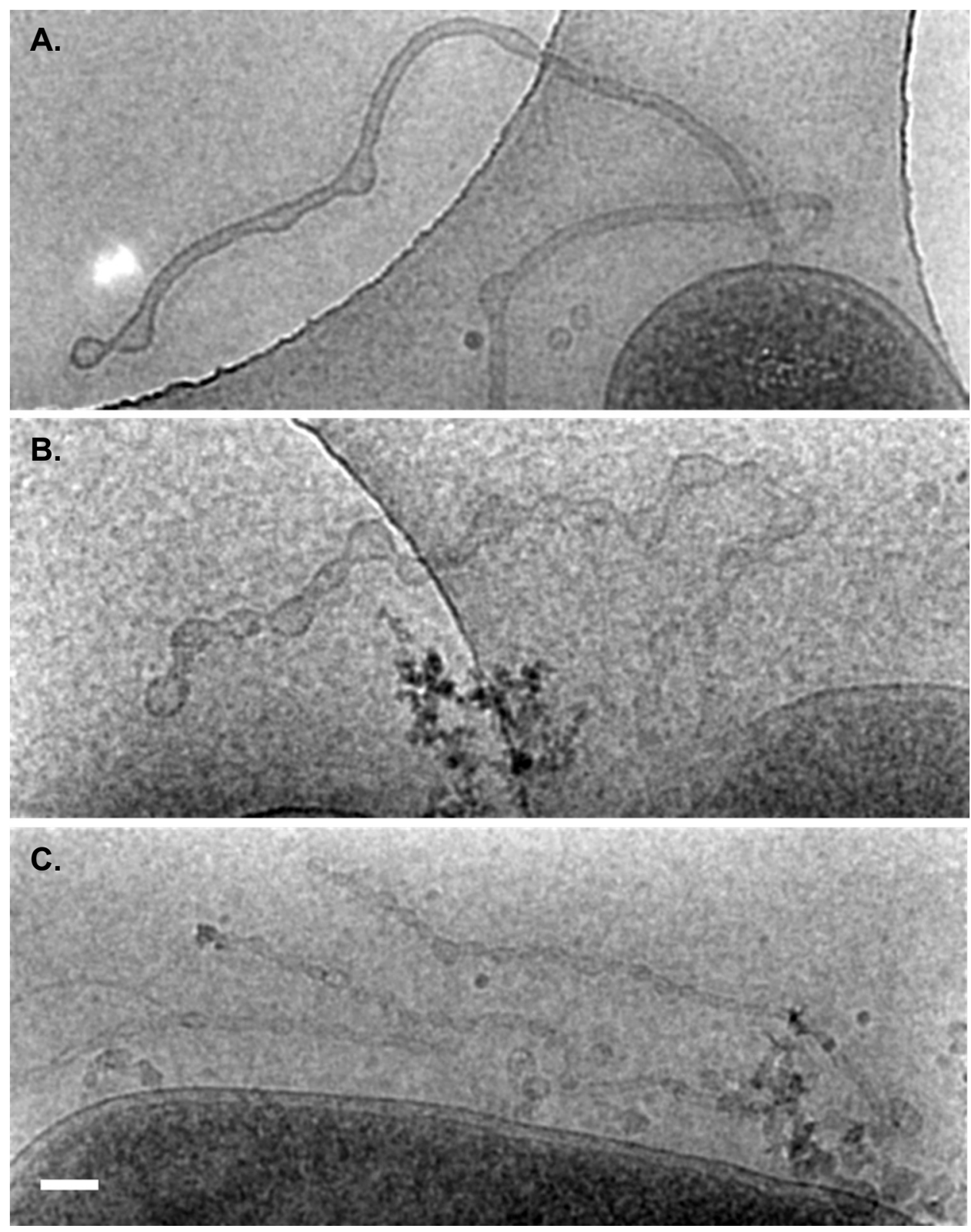

845 Cryo-TEM of $S$. oneidensis WT (A), $\triangle b d p A(\mathrm{~B})$, and $\triangle b d p A$ p452-bdpA (C) OMEs at 90 846 minutes post-surface attachment. Scale $=100 \mathrm{~nm}$. 


\section{Supplemental Figure 4}
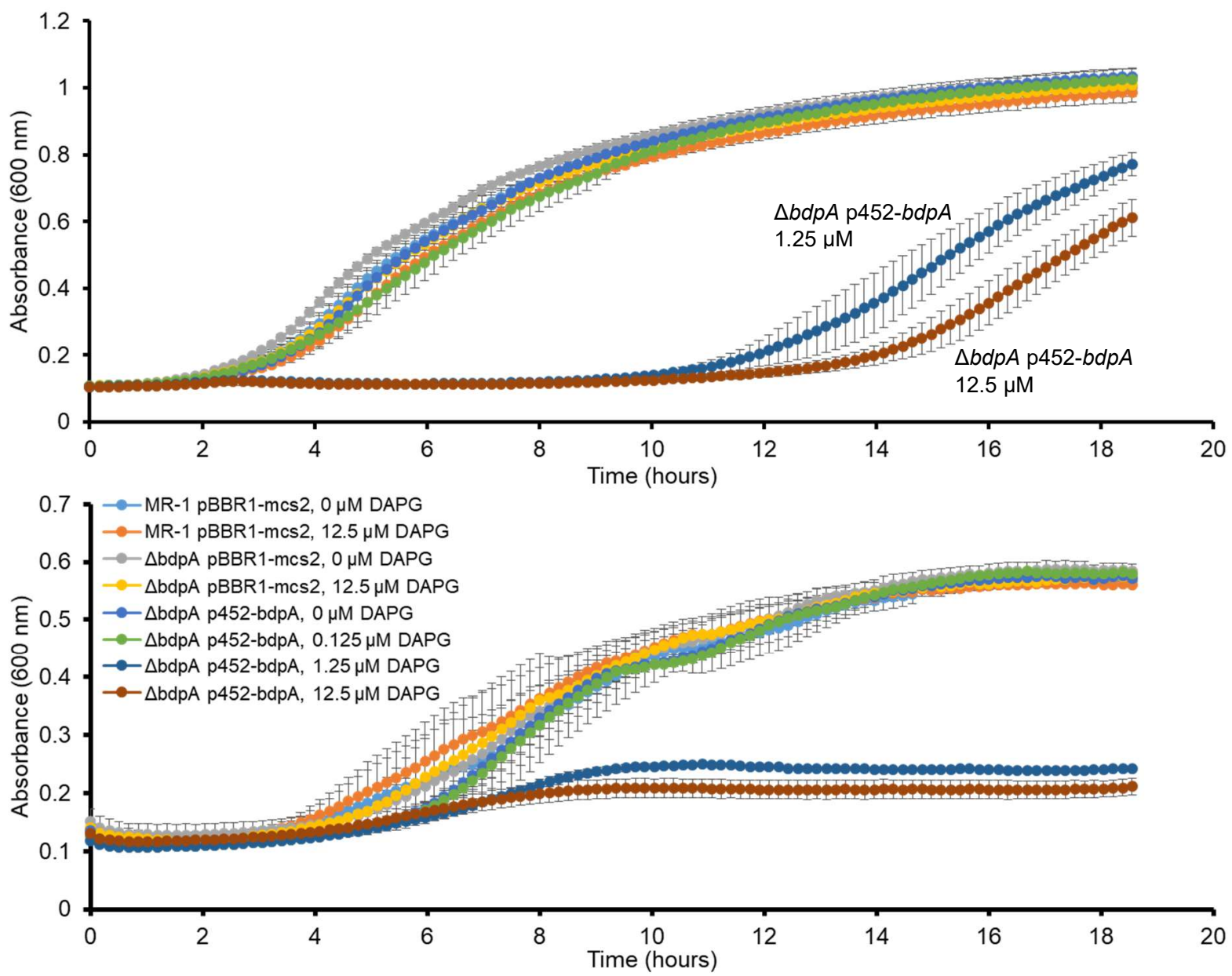

850 Growth of S. oneidensis strains in LB (top) or Shewanella Defined Medium (SDM) 851 (bottom) in response to DAPG exposure and BdpA induction. Error bars are standard 852 deviation of three biological replicates. 


\section{Supplemental Figure 5}
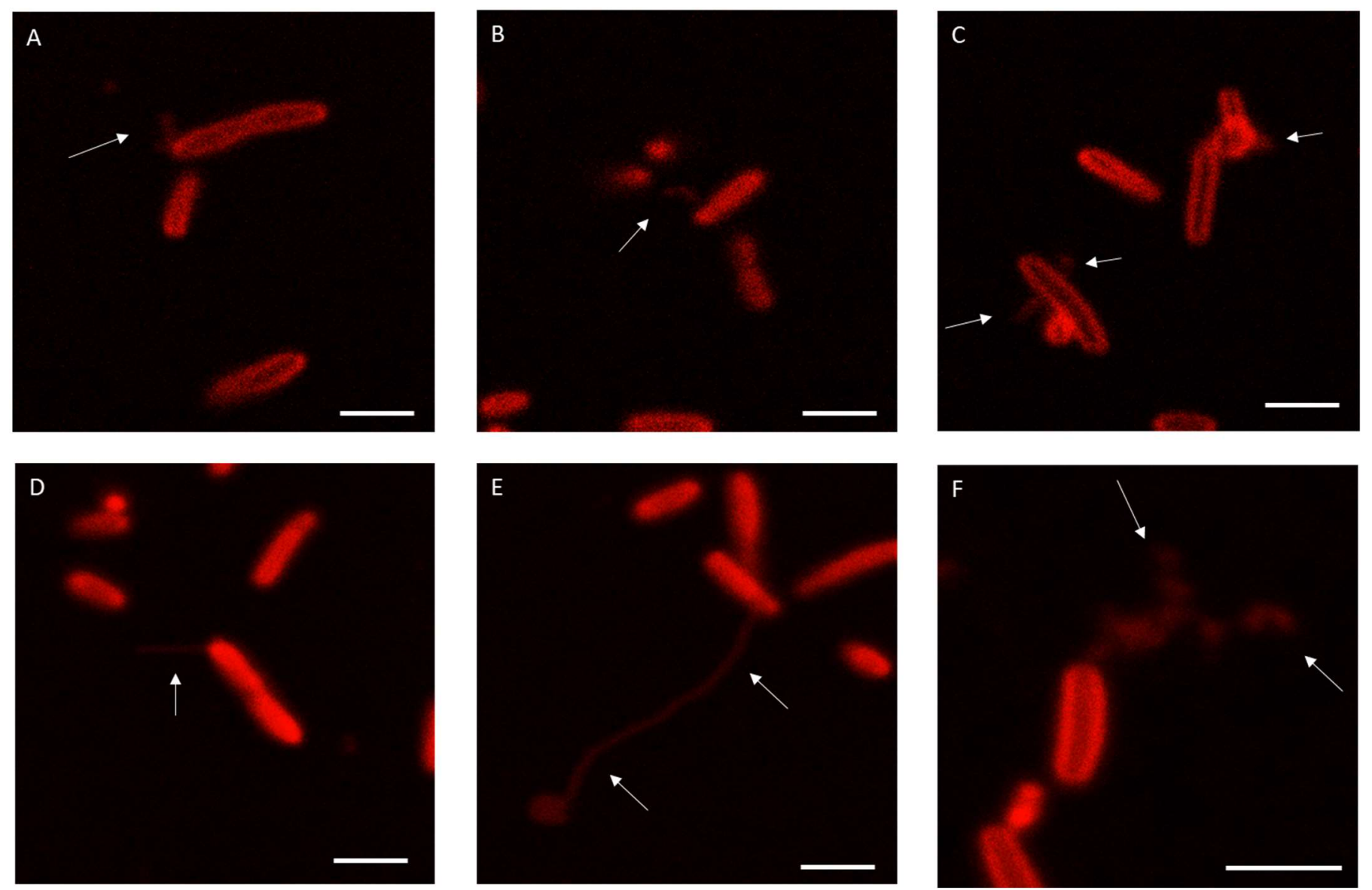

856 Variability in OME phenotypes following BdpA induction in M. atlanticus CP1

857 p452-bdpA cells. Cells displayed an array of membrane curvature phenotypes, ranging 858 from short OMEs $(<2 \mu \mathrm{m}, \mathrm{A}-\mathrm{C})$, long OMEs $(2-10+\mu \mathrm{m}, \mathrm{D}-\mathrm{F})$, membrane blebbing $(\mathrm{C}, \mathrm{F})$, 859 and branched OME/OMV chains $(F)$. Scale $=2 \mu \mathrm{m}$. 


\section{Supplemental Figure 6}

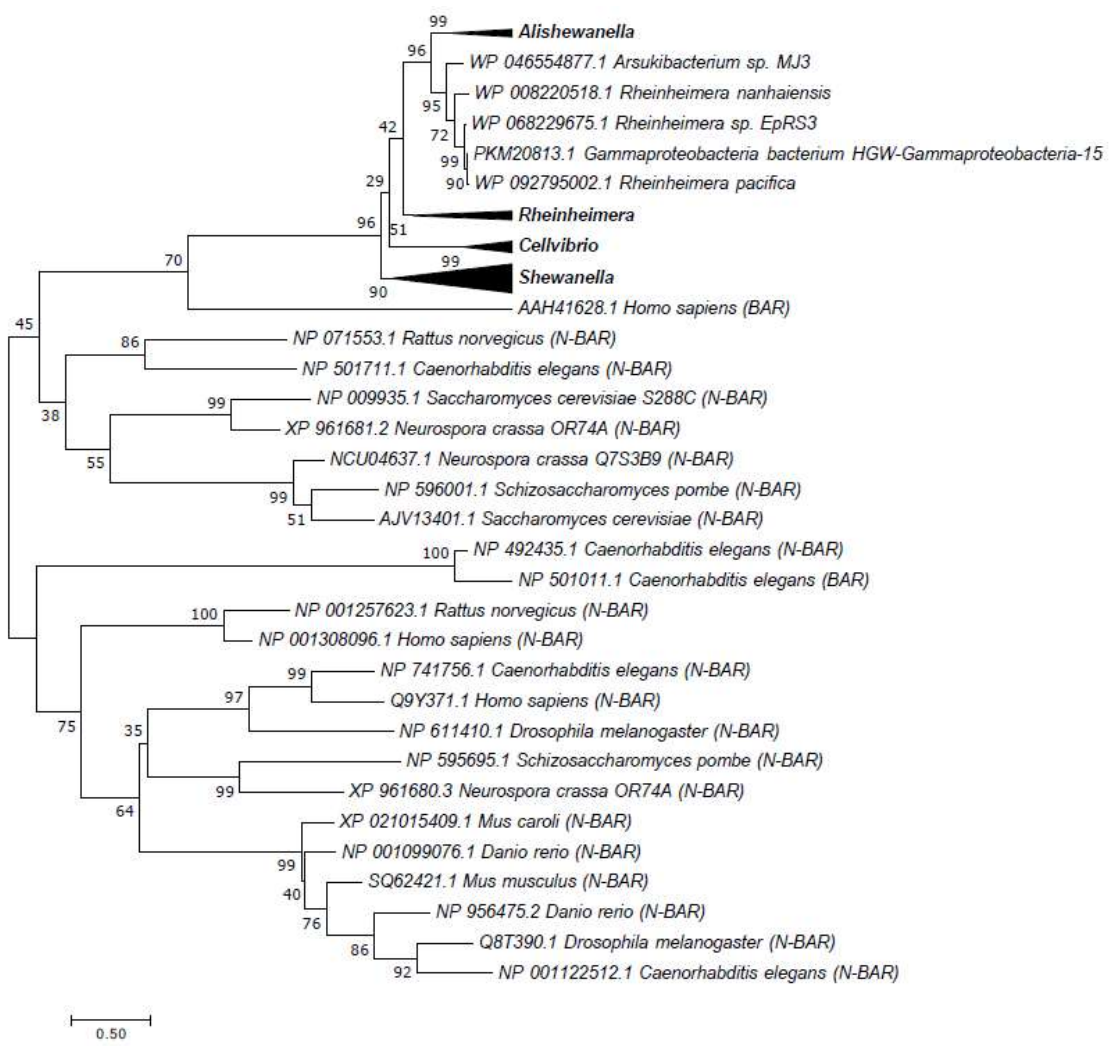

BdpA has homologs in other bacterial species. A phylogenetic tree of the 23 BAR domain sequences that seed the BAR domain HMM predictions, S. oneidensis BdpA, and conserved BdpA orthologs in other prokaryotes. The protein regions corresponding to the BdpA BAR domain sequence from the 52 prokaryotic BdpA orthologs were aligned with the 23 representative eukaryotic BAR domain-containing proteins used to generate the BAR domain consensus sequence (cd07307) at a total of 222 positions. Maximum Likelihood evolutionary histories were inferred from 1000 bootstrap replicates, and the percentage of trees in which the taxa clustered together is shown next to the branches. The Gamma distribution used to model evolutionary rate differences among

872 sites was 11.9548 
874 Supplementary Video 1 Widefield imaging of S. oneidensis WT cells 3 h post-

875 deposition onto the surface of a chambered cover glass. Scale $=5 \mu \mathrm{m}$.

876

877 Supplementary Video 2 Widefield imaging of $S$. oneidensis $\triangle b d p A$ cells $3 \mathrm{~h}$ post-

878 deposition onto the surface of a chambered cover glass. Scale $=5 \mu \mathrm{m}$.

879

880 Supplementary Video 3 Widefield imaging of $S$. oneidensis $\Delta b d p A$ p452-bdpA cells $3 \mathrm{~h}$

881 post-deposition onto the surface of a chambered cover glass. Scale $=5 \mu \mathrm{m}$.

882

883 Supplementary Video 4 Confocal imaging of S. oneidensis MR-1 p452-bdpA cells after 884 1h planktonic induction of BdpA with $12.5 \mu \mathrm{M}$ DAPG. Scale $=5 \mu \mathrm{m}$. 